\title{
L'institution des relations industrielles : le cadre analytique de J. R. Commons
}

Jean-Jacques Gislain

\section{(2) OpenEdition}

Édition électronique

URL : http://journals.openedition.org/ei/720

DOI : $10.4000 /$ ei. 720

ISSN : 2553-1891

Éditeur

Association Économie et Institutions

Édition imprimée

Date de publication : 1 juin 2003

Pagination : 11-59

ISSN : 1775-2329

\section{Référence électronique}

Jean-Jacques Gislain, «L'institution des relations industrielles : le cadre analytique de J. R. Commons

», Économie et institutions [En ligne], 2 | 2003, mis en ligne le 31 janvier 2013, consulté le 19 avril 2019. URL : http://journals.openedition.org/ei/720 ; DOI : 10.4000/ei.720 


\title{
L'institution des relations industrielles : le cadre analytique de J. R. Commons
}

\author{
J.-J. Gislain ${ }^{1}$, Département des relations industrielles de \\ l'Université Laval (Québec), PHARE et CRISES,
}

\begin{abstract}
Dans une contribution précédente nous avons présenté la pertinence du concept de futurité et de ses développements en termes de causalité institutionnelle chez J. R. Commons (Gislain 2002). Dans le présent article nous nous proposons de présenter l'application qui peut être faite de cette construction conceptuelle pour la définition d'un cadre analytique commonsien dans le domaine des relations industrielles; domaine dans lequel J. R. Commons a été très actif comme théoricien et praticien ${ }^{2}$ et dont l'œuvre a été séminale (Barbash 1989; Chasse 1991; Kaufmann 1993, 1998; Hills 1998; Ramstad 1998; Bazzoli 2000a).
\end{abstract}

Il n'est pas facile de présenter ce cadre analytique commonsien. Plusieurs options sont envisageables. L'une d'elles consisterait à faire œuvre d'historien de la pensée économique. Une autre option serait, rétrospectivement, de montrer la pertinence de ce cadre analytique par rapport à l'état actuelle de la connaissance dans le domaine des relations industrielles. Ces deux options sont légitimes et méritaient chacune une contribution spécifique, peutêtre plus d'ailleurs la seconde ${ }^{3}$ que la première ayant déjà fait l'objet d'une littérature abondante ${ }^{4}$. Cependant l'option choisie consiste plutôt à recomposer ce cadre d'analyse, que Commons présente certes de façon cohérente mais de manière éparse dans son œuvre, en le centrant sur le concept pragmatiste et structurant de futurité significative commune. Cette recomposition est d'autant plus légitime que, comme procédé de production de savoir, elle est conforme à l'épistémologie pragmatiste prônée par Commons (Tool 1994). Cette représentation peut aussi avoir le mérite, de par son unité

\footnotetext{
1 Département des relations industrielles de l'Université Laval (Québec), PHARE et CRISES, (jean-jacques.gislain@rlt.ulaval.ca). Je remercie Sylvie Morel et les rapporteurs anonymes pour leur lecture critique attentive et constructive de ce texte. ${ }^{2}$ La bibliographie de J. R. Commons, à la fin de l'article, illustre, par son ampleur, la fécondité de cette œuvre dans ce domaine à l'époque où, aux États-Unis dans le premier tiers du vingtième siècle, émerge le champ d'étude des relations industrielles. ${ }^{3}$ Voir notamment Y. Ramstad (1993), M. H. Rutherford (1994), L. Bazzoli \& T. Kirat \& M-C. Villeval (1994), V. Dutraive (1995), G. M. Hodgson (1998), L. Bazzoli \& T. Kirat (1999) et L. Bazzoli \& V. Dutraive (2002).

4 Voir notamment J. Barbach $(1989,1994)$, J. D. Chasse (1997), B. E. Kaufman (1998), Y. Ramstad (1998), S. M. Hills (1998) et L. Bazzoli (2000a).
} 
d'exposition, de mettre en évidence la pertinence de ce cadre analytique ${ }^{5}$.

Les relations industrielles, ou relations professionnelles selon la terminologie plus restrictive souvent retenue en France ${ }^{6}$, sont un domaine d'étude concernant un objet spécifique de l'activité économique : la relation salariale, le travail et l'emploi et leurs multiples facettes économiques et sociales. En somme, il s'agit d'un domaine transdisciplinaire qui s'intéresse à tout ce qui concerne le monde du travail et qui étudie les multiples aspects de la société salariale.

Selon l'approche commonsienne (1924, 1934a, 1950)7, les acteurs qui vivent dans ce monde sont régis par des institutions ${ }^{8}$ contrôlant leurs activités. Le terme de contrôle signifie la prégnance de modes de pensée et d'agir sur l'action requise ou permise "en société" (concern), c'est-à-dire sur les trans-acteurs dans un monde du travail plus ou moins organisé. Ces institutions et le contrôle qu'elles exercent sur l'action individuelle dans les transactions sont ce que Commons appelle l'action collective. Cette dernière n'est pas réductible à l'action commune d'ensemble d'un collectif ${ }^{9}$. L'action collective, au sens conceptuel de l'approche institutionnaliste commonsienne, s'applique aussi et peut-être surtout aux actions individuelles instituées par/dans un tel collectif. En somme, ce sont toutes les actions individuelles contrôlées par les règles opérantes de conduite (working rules) constitutives de l'action collective. Dans cette optique, les acteurs individuels sont donc socialement construits, ce sont des personnalités instituées pour agir selon des règles opérantes de conduite, ce sont des citoyens d'une société (concern : collectivité, groupement humain, association, entreprise, etc.) en devenir (d'où le gérondif "going" dans going concern) dont les actions sont "sous contrôle" de l'action collective spécifique à cette société en devenir (Atkinson \& Reed 1992).

\footnotetext{
5 Dans son article dans ce numéro, Sylvie Morel se livre aussi à un exercice assez similaire à propos du concept commonsien de "sécurité économique".

${ }^{6}$ Cette acception plus restrictive en France provient sans doute du fait de l'inexistence dans ce pays d'un champ disciplinaire institutionnalisé des "relations industrielles". L'intersection interdisciplinaire ayant pour objet les "relations professionnelles" est en quelque sorte résiduelle par rapport aux sous disciplines institutionnalisées de l'économie, le droit, la sociologie, l'histoire, etc., du travail.

7 Pour une présentation de l'approche commonsienne, voir Y. Ramstad (1990). Pour une excellente présentation générale en français de cette approche, voir L. Bazzoli (2000b).

8 Pour éviter les confusions terminologiques, les concepts commonsiens sont identifiés en italique dans le texte. Dans l'optique de ne pas trop alourdir le texte de références exégétiques aux œuvres de Commons, nous limiterons les références et les citations. Les ouvrages théoriques de Commons $(1924,1934 a, 1950)$ possèdent des index très détaillés auxquels le lecteur intéressé peut se référer.

9 Le terme "action collective" renvoie alors à l'action du collectif en tant qu'entité sociale agissant comme un "individu collectif" (Olson 1966).
} 
Toutefois, une telle approche commonsienne n'est pas intégralement holiste. Elle ne relève pas d'un déterminisme complet dans lequel les actions individuelles seraient totalement ou "en totalité" contraintes par une instance dominante inconsciente ou objective (un esprit, un monde moral, des représentations collectives, des règles de conduite abstraites, des rapports sociaux, etc.). Il s'agit plutôt pour cette approche de prendre analytiquement en compte la réalité de la construction sociale de l'action instituée. Dans le monde du travail, les sujets agissants ne sont pas des être vraiment autonomes. Ce ne sont pas des "individus" simplement dotés de rationalité et de ressources, immergés dans un environnement plus ou moins contraignant et les conduisant à opter pour des choix rationnels ou des actions stratégiques selon les circonstances. Dans ce monde, qui est une réalité sociale structurée et organisée, en d'autres termes institué, les sujets pensants et agissants sont socialisés pour agir selon ce que l'action collective attend d'eux. Leur conduite est socialement construite selon les attentes de leurs transacteurs, selon leur statut d'acteur, leurs (non)droits et (non)devoirs, leurs pouvoirs et responsabilités, leurs immunités et incapacités, leurs sécurités et conformités, leurs vulnérabilités et libertés plus ou moins définis et autorisés dans les diverses formules de transaction; en somme, selon autant de caractéristiques de situations et positions d'acteur dont Commons nous offre une conceptualisation élaborée. Tous ces "selon", qui régissent les conduites, ne sont pas des déterminismes stricts. Ce sont autant de guides comportementaux, de règles opérantes de conduite instituées et en perpétuelle évolution. Ces "selon" font plus que situer une action "individuelle" ayant une logique définie par ailleurs (homo oeconomicus utilitaire et stratégique, acteur cognitif expérimentant et apprenant, etc.). Ils font plus que prendre en compte les "circonstances" dans lesquelles un acteur "individuel", postulé a priori déjà là comme sujet pensant et agissant, s'adapterait en intégrant les "variables" d'un monde extérieur fait de significations et de pratiques à utiliser comme autant de ressources et de compétences. Ces "selon" construisent effectivement la personnalité instituée de l'acteur par/pour sa socialisation dans l'action collective.

L'interactionnisme social et symbolique commonsien qui est mobilisé ici sur le registre de ces "selon", et dont l'orientation théorique de type trans-actionniste s'inscrit dans la lignée pragmatiste de John Dewey et G-H. Mead (Albert \& Ramstad 1997, 1998), est constructif au sens où les acteurs construisent leurs rôles, leur conduite, leur personnalité institués dans/par les transactions. Ce n'est donc pas un interactionnisme de type entre-actionniste où les acteurs adapteraient leur comportement (utilitariste, cognitivisme, etc.) à la co-présence d'autres acteurs pour échanger, se coordonner, s'accorder "entre eux". Pour l'analyse commonsienne, 
il s'agit de prendre acte, en particulier dans l'analyse du monde du travail, de cette réalité sociale prégnante et significative que sont les institutions, c'est-à-dire des actions collectives qui construisent et contrôlent, sans les déterminer totalement, les actions individuelles. Les sujets, pensants et agissants dans ce monde, sont analysés comme des personnalités instituées plus ou moins sous contrôle et, du fait même de la plus ou moins grande incomplétude et instabilité de ce contrôle, générateurs de l'évolution institutionnelle dans ce monde.

La pertinence de la centralité analytique du concept d'institution, et Commons après Veblen (Gislain 2000a) en fut l'un des artisans des plus constructifs, n'est plus maintenant à démontrer avec la renaissance florissante actuellement des multiples néo-post-new-etc. institutionnalismes. Cependant, l'un des aspects analytiques de l'institutionnalisme original, en particulier celui de Commons, a été sous-estimé sinon négligé. Cet aspect est que l'institution, comme concept opératoire de l'analyse institutionnaliste, trouve une bonne part de sa pertinence dans le fait d'intégrer une dimension cruciale de l'activité humaine en société : l'inscription dans une futurité (futurity) socialement construite, dans un à-venir qui est structuré collectivement, une sorte de devenir commun plus ou moins sécurisé pour chacun et qui possède donc des dimensions beaucoup plus riches que celle du simple temps chronologique. Ce sont ces dimensions de la temporalité (timeless) institutionnelle des conduites économiques, d'une futurité plus ou moins structurée par les institutions et au service ou contrôlant l'activité individuelle que nous appellerons la futurité significative commune et que nous présenterons dans un premier temps, et cela dans le cadre spécifique des relations industrielles, c'est-à-dire du monde institutionnalisé du salariat.

Cette présentation générale nous permettra ensuite de mettre en évidence la pertinence du cadre analytique commonsien en matière d'étude des relations industrielles et, tout en conservant l'emphase sur la dimension futurité de l'institution, en adoptant le mode de déclinaison analytique commonsien en termes de transactions de marchandage, de direction et de répartition.

\section{1 - La futurité significative commune}

L'un des apports fondamentaux de la philosophie pragmatiste ${ }^{10}$, en particulier celle de C. S. Peirce (1931-1958) et John Dewey (1967-1990), à l'institutionnalisme commonsien ${ }^{11}$ est la centralité du concept de futurité dans la compréhension des

10 Sur la philosophie pragmatiste, voir D. Deledalle (1954) et J. P. Diggins (1994).

${ }^{11}$ Sur l'influence de la philosophie pragmatiste sur Commons, voir Y. Ramstad (1986),

P. Mirowski (1987), A. Albert \& Y. Ramstad $(1997,1998)$ et M. Renault (2000). 
conduites humaines. En effet, comme nous l'avons développé ailleurs (Gislain 2000b, 2002), le pragmatisme offre à la compréhension de la conduite humaine un fondement important. Il consiste à considérer que le sujet pensant et agissant ne vit pas dans un monde atemporel ou simplement chronologique. L'une des caractéristiques fondamentales de l'esprit et du comportement humains est de se projeter dans l'avenir, d'être une volonté de penser et d'agir inscrite dans un devenir. Pour se faire, n'est appréhendé comme vérité et réalité que ce à quoi il est raisonnablement possible de s'attendre. Ce sont les croyances dans le domaine de la pensée et les habitudes dans le domaine de l'agir qui permettent à chacun de contrôler le/son monde et son avenir dans celui-ci. Ainsi, la célèbre formule de C. S. Peirce, reprise par Commons : "Considérer quels sont les effets pratiques que nous pensons pouvoir être produits par l'objet de notre conception. La conception de tous ces effets est la conception complète de l'objet " (Peirce 1879, 297; Commons 1934a, 152), et appliquée à la conduite humaine, conduit au " principe de futurité [selon lequel] les hommes vivent dans le futur mais agissent dans le présent "(Commons 1934a, 84). Selon ce principe, à savoir que le " Pragmatisme est Futurité "(Commons 1934a, 152), la conduite humaine, dans les domaines de la pensée et de l'action qui ne peuvent d'ailleurs être séparés, est déterminée et ne peut être comprise qu'en rapport aussi, et peut-être surtout, à un monde qui n'est ni celui purement objectif de l'environnement extra individuel, c'est-à-dire le monde de la nature extérieure au sujet, ni celui purement subjectif de l'intériorité mentale individuelle et singulière, c'est-à-dire le monde de la nature intérieure au sujet.

Ce "troisième monde", que propose la philosophie pragmatiste pour dépasser la logique cartésienne sans temporalité spécifiquement humaine et articulée sur le dualiste des deux "mondes" de l'extériorité objective / intériorité subjective, est en quelque sorte un monde medium entre les deux mondes cartésiens et un monde futuristique (futuristic) par rapport à eux. Ce troisième monde, mis en évidence par la philosophie pragmatiste pour appréhender les spécificités de la condition humaine, est précisément celui du lieu et de la temporalité propres à la signification et à la futurité. Ce troisième monde, toujours contingent et en évolution, en quelque sorte un monde ambulatoire selon les exigences et aléas de la condition humaine, est celui dans lequel pense vivre réellement et "à preuve du contraire" le sujet pensant et agissant. Ce monde est celui dans lequel ce sujet déploie sa volonté de devenir selon ses croyances avérées sur ce qu'est pour lui la réalité présente et à-venir et selon ses habitudes comportementales valables et durables autant qu'elles ne sont pas infirmées par l'expérience. En somme, ce sujet se conduit selon ce qu'il peut raisonnablement attendre du monde, c'est-à-dire selon les "sens" de 
sa pensée/signification et de son action/orientation. Dès lors, la conduite de ce sujet est déterminée par un principe de causalité dont le sens est "inverse" de celui du monde physique allant du passé vers le présent. Il s'agit d'un principe de causalité allant de la futurité/cause vers le présent/effet.

Ce monde "pragmatique" de la futurité, habité de sens pour penser (significations) et pour agir (orientations), n'est pas un lieu dont la dimension individuelle singulière nous intéresse ici : le fait que chacun possède son troisième monde propre à soi, habité de croyances et d'habitudes singulières qui aident à vivre "malgré" autrui et l'environnement, c'est-à-dire le fait de penser et d'agir de façon "autonome" selon ses propres conduites singulières quasi autistiques. La dimension de ce troisième monde qui nous intéresse ici, et qui est sans doute le plus important pour la vie en société, est celui commun des attentes partagées quant à ce qui est plus ou moins "correct" de penser et de faire une société de destins partagés (going concern). Ce monde est donc celui de la futurité significative commune, celui du devenir en commun et ayant des sens partagés, celui de l'inscription dans le champ de la socialité comme projet "en société". Ce monde, dans lequel est donc construit le lien social comme avenir en commun, est ainsi celui des institutions, au sens précis du concept d'institution proposé par Commons dans la lignée de la philosophie pragmatiste, c'est-à-dire un monde du vivre ensemble, de l'action collective, de l'ensemble des significations et des règles opérantes de conduite qui offre à chacun, et/car en concert avec autrui, un avenir envisageable.

Ce monde qui permet le vivre ensemble selon un projet de "sens communs" (sens / signification-pensée; sens / orientationaction) et qui fonde le principe de causalité institutionnelle (ces "sens" de la futurité significative commune déterminant l'action collective contrôlant l'action individuelle présente), ne requiert pas analytiquement d'être fondé sur une quelconque métaphysique transcendantale unificatrice du sens de la vie en commun, ni sur une quelconque philosophie d'un principe supérieur commun fondé en Raison, en Justice ou relevant d'une quelconque autre instance de justification du vivre en accord avec autrui. Ce monde résulte plus prosaïquement d'un état de fait historique que les sujets pensants et agissants en société trouvent déjà là en naissant et dont l'expérience et l'apprentissage (" Mouche ton nez et dit bonjour à la dame !") leur ont très vite appris qu'il était une condition nécessaire (au risque de l'autisme social ou de l'exclusion) et plus ou moins obligée (au risque de sanction) de la vie "en société". En somme, ce monde est celui qu'abordent traditionnellement les sciences sociales en termes de construction sociale des acteurs et que l'approche commonsienne peuple d'esprit institué, de personnalité d'acteur institué comme trans-acteur ou encore de citoyen d'un groupement 
humain en devenir (going concern). La généalogie d'un tel monde des institutions, d'une futurité significative commune fournissant à chaque être social un appareillage sensé (signification) et orienté (action) de conduites ("sous contrôle" de l'action collective et plus ou moins empreintes de domination) du vivre-ensemble, reste encore à découvrir et demeure, il faut bien l'admettre, quelque peu énigmatique. A cet égard donc, la tradition rationaliste de la recherche des fondements premiers du lien social est analytiquement contre productive, si ce n'est pour l'alimentation idéologique de justifications nourrissant la légitimé actuelle de la futurité significative commune en termes d'accords de coordination plus moins coopérative, de compromis plus ou moins négociés ou consensuels, de contrats et d'équilibres plus moins optimaux, etc., entre "individus". En revanche, l'approche institutionnaliste, en particulier commonsienne, assume comme point de départ analytique réaliste, c'est-à-dire sans contenu normatif $a$ priori sur le fondement premier in abstracto du lien social, la prégnance concrète, le matter of fact des institutions, c'est-à-dire la "réalité", dans ses manifestations sociales concrètes, de ce troisième monde de la futurité significative commune rendant possible les conduites en société. Pour cette approche, c'est donc uniquement ce possible constaté, le fait qu'il y ait effectivement des institutions opératoires du lien social, et en particulier grâce à leur dimension de futurité significative commune, qu'il s'agit d'analyser "réalistement".

\section{1 - La futurité significative commune dans le monde du travail}

Dans le monde du travail, qui est l'objet d'étude des relations industrielles, ce troisième monde de la futurité significative commune, fait de significations et de règles opérantes de conduite permettant à chacun de s'inscrire dans le devenir de la société salariale, est plus prégnant que dans d'autres dimensions de l'activité économique. La raison en est sans doute que, dans notre civilisation occidentale contemporaine, la relation salariale est un haut lieu de socialité structurée selon un registre de face à face et s'inscrivant dans une durée de projet en commun. Contrairement aux apparences d'impersonnalité et d'instantanéité d'autres activités économiques, l'acte d'achat/vente d'une marchandise par exemple, la relation salariale est un rapport social explicite, une relation économique qui implique une relation de personne à personne et un engagement dans la durée. Cette caractéristique de la relation salariale requiert, plus que dans d'autres relations économiques plus ponctuelles et médiées par un opérateur abstrait à signification apparemment moins équivoque, une relation monétaire à l'occasion de l'achat/vente d'une marchandise standardisée par exemple, de la part des acteurs de la relation salariale une démarche de clarification 
(signification commune) et de mise en perspective (futurité) de ce que sera, pour chaque sujet pensant et agissant, "travailler" ... "contre" un futur salaire et autres avantages, "pour" un employeur et sa hiérarchie organisationnelle, "avec" des collègues et "dans" un collectif de travail, "selon" certaines méthodes de travail et de production, etc., en d'autres termes d'accéder à ce troisième monde de la futurité commune et signifiante qu'est le "monde du travail". De plus, ce monde n'a pas l'apparence d'harmonie sociale que peuvent présenter d'autres formes d'activité économique, l'apparence d'équivalence ou l'équilibre dans la relation monétaire marchande par exemple. Ce monde est un lieu où le conflit est toujours présent ou latent, un lieu où se confrontent perpétuellement et "en personne" les attentes et leurs significations, un lieu donc où il est nécessaire, pour continuer à vivre ensemble dans un projet durable, de faire des compromis, de négocier des ententes, de s'accorder sur des attentes communes et temporaires, sur ce que seront les salaires, les charges et bénéfices de chacun, les définitions de tâche et de charge de travail, les subordinations, les pouvoirs et les responsabilités, les droits et les devoirs de chacun, etc., en somme un ensemble de modus vivendi et de modus operendi suffisamment raisonnable pour que chacun accepte plus ou moins de "travailler ensemble dans le futur".

Ce monde du travail, structuré selon une variété de ce type de modus vivendi et modus operendi et perpétué par le troisième monde de la futurité significative commune des trans-acteurs de la relation salariale, n'est pas un monde uniforme fait d'interdépendances entre acteurs à statut équivalent, égalitaire dans des relations réciproques d'échange comme le propose la fiction d'un autre troisième monde qui serait celui du "marché" : un monde atomisé d'homo œeconomicus sans lien durable, sans temporalité sociale et sans autre signification partagée que l'intérêt individuel bien compris et conclu dans des contrats optimaux. Le monde du travail est un monde de relations de subordination, sinon de domination et de coercition, et de relations entre groupes sociaux plus ou moins organisés et représentés. Cette autre caractéristique est aussi d'une grande importance pour les composants de la construction du troisième monde de la futurité significative commune qui permet le devenir ensemble des acteurs de la relation salariale. En effet, ce monde, peut-être plus que tout autre dans l'activité économique, est un monde politique, au sens où les relations y sont construites selon des logiques de lutte/cohésion allant de l'affrontement entre acteurs antagoniques (salariés-syndicat vs employeurs-patronat) jusqu'à la légitimation-justification de l'autorité-pouvoir de dire ce que seront les conduites acceptables. L'enjeu de la recherche de puissance pour les acteurs individuels ou collectifs en conflit/partenariat, selon des modalités allant du rapport de forces à la négociation raisonnée, est la définition du 
régime futur de la vie en commun dans le monde du travail, c'est-àdire de la futurité significative commune sur laquelle les adversaires/partenaires s'entendent ou non, élaborent ou non un armistice/compromis plus ou moins précaire dans son contenu et dans le temps. Les concrétisations résultant de ce rapport politique et qui structureront la futurité significative commune des acteurs de la relation salariale pour un certain temps, et dont les conventions collectives et autres formes législatives, juridiques ou réglementaires sont l'expression la plus formelle, expriment clairement et concrètement ce que sont les institutions dans le monde du travail : ce à quoi chacun doit(pas)/peut(pas)/pourrait(pas) raisonnablement s'attendre dans l'avenir, c'est-à-dire quelles seront les situations, positions, significations correctes ou non des acteurs et leurs actions définies dans les diverses formules de transaction constitutives du monde du travail. Ainsi, ce monde, s'il n'est pas exempt de certaines formes d'autonomie permise des acteurs, de contingence radicale de certaines situations, de certains phénomènes émergents non intentionnels et éventuellement de comportements stratégiques de certains acteurs dotés de pouvoirs/libertés, il est cependant le plus fréquemment et le plus communément régi par une futurité significative commune très structurée, par un à-venir conditionné par un ensemble de règles opérantes de conduite plus ou moins organisées, ce que Commons appelle l'action collective : ce à quoi chacun doit/peut/pourrait plus ou moins se conformer pour s'inscrire dans un projet commun de production sous un régime de travail salarié.

Ce dernier aspect du monde du travail, comme mode d'activité en commun et comme construction sociale particulièrement structurés dans la durée par ce troisième monde de la futurité significative commune, explique pour une bonne part pourquoi le problème de la coordination des acteurs est pour l'essentiel un faux problème formel découlant logiquement de l'hypothèse a priori de l'autonomie première de l'acteur (que ce soit un simple homo œeconomicus "idiot" ou, plus sophistiqué, un être cognitif "intelligent"). Dans le monde du travail, qui existait avant et qui existera après l'apparition d'un acteur qui y vit pour un temps comme personnalité instituée, la durée des institutions désingularise en quelque sorte 1"individu", le formate s'il on peut dire, non selon une logique purement déterministe en en faisant un automate, mais, au contraire, en le personnalisant comme esprit institué, comme sujet pensant et agissant selon des rôles de composition, ceux que le troisième monde de la futurité significative commune lui offre, sans qu'il ait d'ailleurs beaucoup le choix de les refuser, pour pouvoir agir (power of action) en concert avec autrui. La prégnance, le contrôle, exercés par l'action collective structurée, selon les éléments de la futurité significative commune, sur l'action 
individuelle dans le monde du travail est aussi, et peut-être surtout, une dotation ou distribution institutionnalisée de (non)pouvoirs, de (non)libertés, de (non)opportunités, de (non)sécurités, de (non)droits et (non)devoirs, etc. En somme, la contrainte qui pèse sur l'action instituée dans le monde du travail n'est pas seulement une contrainte purement et négativement "contraignante", c'est-à-dire aliénante au sens d'une perte ontologique de la maîtrise par l'acteur de ce qui serait pour lui en soi de bonnes raisons de penser et d'agir, elle est aussi, et peut-être surtout, une contrainte positive de socialisation, une contrainte plus ou moins intégrée d'inscription dans un projet commun et concerté de production, dans un à-venir possible avec autrui dans le monde du travail; en somme, ce qu'il y a de requis comme condition nécessaire, comme "bon sens" (signification/orientation) pour une coopération productive plus ou moins durable et efficace. A cet égard, dans le monde du travail en particulier, le postulat méthodologique de pur opportunisme de l'action stratégique de type utilitaire est incompatible avec la compréhension d'une possible action durable en commun. Si la contrainte est comprise comme une simple donnée environnementale ou circonstancielle de l'action autonome, stratégique et utilitaire, alors le monde du travail est tout simplement impensable car précisément impensé (insensé) - absence du troisième monde de la futurité significative commune - par (pour) les acteurs ... incapables bien évidemment de se coordonner faute d'avoir été socialisés comme acteur institué dans un projet en commun s'inscrivant dans la durée.

Pour l'essentiel, en ce qui concerne l'activité humaine dans le monde du travail, les règles opérantes de conduite, c'est-à-dire l'action collective contrôlante et la futurité signifiante commune qui la projette dans la durée, sont déjà là; il n'est pas sans cesse autorisé, même si comme nous le verrons plus bas certains événements le permettent sinon le nécessitent, de discuter les modus vivendi et modus operendi établis, de "jouer" sur/avec/contre les contraintes de l'action collective. En somme, ce que d'aucun après Max Weber appelle la bureaucratie et ce que Commons nomme, de façon générale et sans connotation normative quant à sa rationalité, l'action collective est justement cette dimension, certes contraignante, mais (souvent tristement) nécessaire à l'action "en concert" dans le monde du travail en régime salarial.

Par ailleurs, que cette dimension contraignante de l'action instituée soit plus ou moins empreinte de subordination, sinon de domination et de coercition, pour la plupart des salariés - ce qui fonde, entre autre, pour Commons (1935), la connexion incontournable entre économie, droit et éthique ${ }^{12}-$, ne signifie pas

${ }^{12}$ Sur cette connexion, voir W. M. Dugger (1980), S. G. Medema (1998) et L. Bazzoli et T. Kirat (1999). 
pour autant qu'elle soit éliminable, parce que condamnable d'un quelconque point de vue relatif à la justice. La question pertinente par rapport à la contrainte, dès lors que le salariat n'est pas remis en question comme régime dominant le monde du travail, est celle de son caractère plus ou moins raisonnable. La contrainte doit être compatible avec la double exigence, d'une part, d'ordre (propriété), d'efficacité (coopération) et de gouvernance (souveraineté) économiques, d'autre part, de justice (droit/éthique) limitant la coercition et la subordination abusive et donc le conflit ouvert. C'est cette question incontournable du raisonnable (reasonabless, celle plus générale du politique dans la littérature organisationnelle nordaméricaine dans la tradition Parsons-Dunlop) qu'aborde aussi Commons quant à la dimension normative acceptable et pas seulement fonctionnelle-intégrative de l'action collective. Dans le monde du travail, la futurité significative commune des trans-acteurs est certes fonctionnellement contraignante, en particulier pour les travailleurs du fait même de la forme salariale de l'activité laborieuse, mais elle doit aussi être raisonnablement acceptable, c'est-à-dire avoir un sens (signification / orientation) supportable et endurable pour les salariés.

\section{2 - L'évolution de la futurité significative commune}

Une autre caractéristique importante de ce troisième monde de la futurité significative commune régissant les perspectives d'activité dans le monde du travail est qu'il est le lieu de la genèse et de l'évolution des institutions de la société salariale. Analytiquement, il est donc nécessaire pour Commons, dans une optique évolutionnaire inhérente à l'approche institutionnaliste ${ }^{13}$, de rendre compréhensible pourquoi et comment la relation salariale évolue.

En effet, bien que la dimension contraignante de l'action collective soit essentielle à l'activité "en concert", il n'en demeure pas moins que deux aspects importants du monde du travail impliquent une sorte de nécessité quant à l'évolution des institutions du salariat. L'un de ces aspects est l'émergence ou la résurgence de conflits qui avaient été temporairement écartés, qui avaient donné lieu à des ententes provisoires ou qui n'avaient pas encore été identifiés par les transacteurs de la relation salariale. Est alors remis en cause ce que signifie travailler en commun selon un projet s'inscrivant dans la durée, c'est-à-dire quelles sont les structures encore acceptables ou nouvelles de la futurité significative commune. L'autre aspect est la contrainte d'adaptabilité et donc de production de nouvelles solutions face à l'existence de situations nouvelles ou non encore régies par le compromis en cours sur la futurité

13 Sur l'approche évolutionnaire de Commons, voir J. E. Biddle (1990), Y. Ramstad (1994) et J-J. Gislain (1999). 
significative commune. Cette contrainte apparaît à l'occasion de l'apparition de conditions environnementales nouvelles, de transformations et d'innovations dans le monde du travail, et en général dans l'activité économique, qui s'imposent comme incontournables ${ }^{14}$. Confrontée à l'existence incontournable du conflit et/ou à la nécessité du changement dans un monde du travail en perpétuelle transformation, la question centrale pour l'analyse commonsienne est alors de savoir quel est le processus institutionnel qui opère l'évolution dans le monde travail, quels sont les processus de dé-re-structuration de la futurité significative commune, ou, pour reprendre la terminologie darwinienne datée de Commons, quels sont les processus de "sélection artificielle" des institutions de la société salariale ? La modalité de réponse principale à cette question, proposée par Commons, est d'analyser le processus institutionnel suivant lequel, un événement-problème étant posé concernant un élément constitutif ou absent de la futurité significative commune en cours d'opération, une autorité, plus ou moins autorisée, autorise une solution-évolution institutionnelle, c'est-à-dire valide ou non un nouvel élément constitutif de la futurité significative commune, en permettant ainsi de restructurer un modus vivendi opératoire dans le monde du travail. Reprenons plus en détails ces points successifs de l'analyse commonsienne de l'évolution institutionnelle du monde du travail car ils sont au cœur de sa conception des relations industrielles.

Un conflit, une inadaptation à la nouveauté, une innovation proposée ou la vacance constatée d'un élément de la futurité significative commune entraînent un événement-problème pour lequel une solution est requise de manière à perpétuer l'opérationnalité de l'action collective supportant le projet concerté de vie au travail en régime salarial. Dans cette perspective évolutionnaire, la relation salariale est donc sans cesse convoquée à se transformer au gré des conflits et innovations qui émergent à l'initiative des trans-acteurs. Ces derniers sont toujours plus ou moins bien institués car les positions et les situations d'acteurs sont toujours plus ou moins contingentes (conflit latent, incomplétude de l'action collective, etc.) et instables (historicité du fait social, innovations, transformations environnementales, et.). En somme, ces évènements-problèmes manifestent certaines pertes de contrôle de

14 A cet égard, l'article de 1909 de J. R. Commons, sur l'influence de l'évolution historique des formes successives de compétition économique sur l'évolution des formes organisationnelles de la production dans l'industrie de la chaussure, est remarquable. Les articles dans ce numéro de J. D. Chasse, sur l'actualité du concept commonsien de "menace concurrentielle" pour comprendre l'état actuel des relations industrielles, et de R. McIntyre \& Y. Ramstad, sur les droits internationaux du travail requis par la globalisation actuelle, s'inscrivent précisément dans le cadre de la problématique commonsienne de l'évolution de la relation salariale convoquée par les faits économiques contemporains. 
l'action collective organisée (de règles opérantes de conduite formelles) ou inorganisée (de règles opérantes de conduite fondées sur des pratiques habituelles). Ces évènements-problèmes sont des déstructurations ou des vacances à combler de la futurité significative commune anciennement opérationnelle. Ces évènements-problèmes peuvent être le fait de nouvelles conditions objectives qui s'imposent de l'extérieur comme de nouvelles conditions environnementales du monde du travail mais ils sont aussi le fait d'initiatives de la part des transacteurs de la relation salariale, en particulier sous les formes du conflit et de l'innovation, de la remise en cause et de l'alternative novatrice, de la révision de règles opérantes de conduite routinières ou de l'apparition d'actions stratégiques. Dans l'approche commonsienne, l'amorce du processus d'évolution de la relation salariale est donc initialement le fait, pour une bonne part, d'actions individuelles. Ces dernières peuvent prendre un caractère de groupe lorsque un évènement-problème concerne plusieurs trans-acteurs mais dans tous les cas, action individuelle ou action de groupe, elles se confrontent à l'ordre établi de l'institution salariale.

Convoquée par ces évènements-problèmes à évoluer et de telle sorte à maintenir une régulation adéquate de la relation salariale, l'institution salariale connaît un processus de résolution de problème (problem solving). Ce processus opère la sélection d'une variation-solution permettant la perpétuation d'une futurité significative commune ainsi dé-re-structurée. Chaque variationsolution sélectionnée est ainsi une nouvelle règle opérante de l'action collective (re)structurant l'institution salariale. Mais cette sélection n'est pas une sélection "naturelle", au sens darwinien par exemple, ou un fait "émergent" non intentionnel, au sens hayekien par exemple. Le processus de sélection requiert des opérateurs agissant comme sélecteurs artificiels. Ceux-ci, dans l'optique de la "sélection artificielle" des institutions défendue par Commons, sont des autorités, personnalités ou instances, qui autorisent les variations devenant ainsi solutions. Ces dernières peuvent être acceptées comme résolutions temporaires de conflit ou subies comme nouvelles contraintes coercitives par les transacteurs de la relation salariale. Le rôle des personnalités ou instances autorisées est ainsi d'autoriser ou non une possible variation-solution, de dire quelle sera la règle opérante de conduite à venir. Ces opérateurs, dont le pouvoir d'autorisation aura donc pour effet de modifier certains éléments de la formule de transaction, et ainsi de modifier la structure de l'action collective en transformant, ajoutant ou supprimant certaines règles opérantes de conduite, peuvent avoir différentes caractéristiques selon l'événement-problème en question et selon le type de légitimité requise, permise, acquise ou imposée de l'autorité autorisée. 
Ces autorités possèdent un pouvoir d'autorisation de variations-solutions : soit parce que ce pouvoir leur a été conféré par une légitimité interne (compétence, notoriété, représentativité, etc.) ou externe (loi, règlement, juridiction, arbitrage, commission, instance paritaire, etc.) au going concern productif; soit parce qu'elles sont l'expression exécutoire d'une négociation, concertation, conciliation, participation, etc., entre trans-acteurs et/ou leurs représentants respectifs (convention collective, accord cadre national, local ou sectoriel, accord d'entreprise, accord participatif, etc.); soit encore, et dans ce cas le conflit n'est pas résolu mais jugulé par la contrainte coercitive, parce qu'elles disposent d'un pouvoir d'autorisation discrétionnaire ou/et arbitraire résultant d'un rapport de forces favorable, individuel ou collectif, pour l'un des "individus" ou "partenaires sociaux" de la transaction (employeur putatif vs salarié candidat, supérieur vs subalterne, gouvernant vs citoyen).

En allant de la forme la plus autoritaire et arbitraire à la forme la plus démocratique ou légitime en passant par l'aspect le plus informel, ces opérateurs, possédant le pouvoir d'autoriser les dé-re-structuration de la futurité significative commune des transacteurs de l'institution salariale, peuvent être : le pouvoir discrétionnaire patronal affirmant, via sa hiérarchie organisationnelle, sa puissance de coercition-dominationsubordination par un diktat sur la règle à venir; le règlement en cour de justice, en procédure d'arbitrage ou de conciliation, par lequel le juge, l'arbitre ou le conciliateur tranche le grief en énonçant la règle jurisprudentielle; le législateur qui réglemente sur la base de la prise en compte de l'avis des partenaires sociaux; l'accord signé sous forme de convention collective, d'accord d'entreprise, de lettre d'entente, etc., entre syndicat et employeur; la personne ou l'instance compétente qui, au sein de la hiérarchie organisationnelle, propose et voit plus ou moins acceptée par le collectif de travail la nouvelle règle opérante de conduite; le collectif de travail lui-même qui, via une représentation, une délégation ou une reconnaissance d'estime ou de compétence attribuée à l'un de ses membres, se donne de nouvelles règles opérantes de conduite.

Dans tous les cas, qu'elles soient plus ou moins légitimes ou arbitraires, négociées ou imposées, fruits d'un partenariat, d'une concertation ou d'une confrontation de rapport de forces, justifiées par une compétence reconnue ou faits iniques, établies par un consensus collectif ou le fait d'une individualité fortement reconnue, expressions de la domination ou de la démocratie industrielle, l'autorité de l'opérateur de la sélection et la nouvelle règle qu'il autorise ou non relèvent, et sont analysables comme telles, d'un processus réel de construction sociale institutionnalisée de la futurité significative commune des transacteurs de la relation salariale. Dans ce processus bien concret et dont les acteurs du monde du travail 
prennent plus ou moins connaissance selon leur degré d'implication requis ou permis par ce processus, il n'y a là rien de bien secret, rien qui ne ressemble à la "rationalité" abstraite d'un "contrat optimal" ou à l'immanence d'un "fait émergent non intentionnel"; l'autorité qui tranche, celle qui a le pouvoir d'autorisation, qu'elles soient une personne, une instance, un résultat de négociation ou un fait de réputation, est bien identifiable et c'est la raison pour laquelle Commons qualifie ce processus de sélection "artificielle". De plus, bien que la nouvelle règle opérante de conduite soit, à l'issue de ce processus, autorisée et comme telle s'impose aux acteurs concernés comme nouveau contrôle de l'action collective, rien ne permet d'affirmer, quoi que cela puisse être le cas parfois, qu'il s'agisse nécessairement d'un "accord" au sens de l'entre-actionnisme évoqué plus haut. Il appert bien souvent que cette autorité résulte d'un fait de pouvoir, quelle est l'expression d'une puissance, quelle traduise un état de dissymétrie où la domination, sous forme de subordination ou de coercition, est bien souvent présente dans le "rapport" salarial.

Ainsi, selon l'approche commonsienne, le champ des relations industrielles est une réalité faite de rapports sociaux où l'autorité, au sens présenté plus haut de pouvoir exprimant sa puissance dans l'autorisation des règles opérantes de conduite structurant la futurité significative commune, joue un rôle crucial avec son cortège d'arbitraire, de coercition, de domination, de subordination, de partenariat, de concertation, de négociation, de conflit, de rapport de forces, de légitimation, de reconnaissance de droits et de compétences, d'imposition de devoirs et d'obligations, etc. Ce champ social n'est donc pas réductible à un simple entre-actionnisme stratégique suivant lequel le "jeu" des acteurs individuels, plus ou moins autonomes et rationnels, conduirait à l'établissement de "règles d'accord" (conventionnelles, co-régulée, etc.) permettant la "coordination" contractuelle dans la relation salariale selon une optimalité réciproque d'intérêts bien compris. Le monde du travail, celui du champ social des relations industrielles, est un monde fortement institué par une futurité significative commune, certes pacifiant ce champ mais toujours lieu de conflits, et en particulier sur qui, la personne ou l'instance, a l'autorité de dire la règle ${ }^{15}$. La question du pouvoir et celle de sa forme crédible de domination qu'est la puissance de dire les règles opérantes de conduite constitutives du régime du salariat, c'est-à-dire la question de qui détermine la futurité significative commune qui contrôlera la vie des

$15 \mathrm{Si}$, comme le met en évidence le philosophe pragmatiste J. L. Austin (1962) " dire c'est faire ", il y a bien un lieu où le discours autorisé est particulièrement performatif, c'est celui du monde du travail, un monde fait d'ordres énoncés et d'obéissance aux règles énoncées par les autorités autorisées. 
salariés, sont au centre du monde du travail. En conséquence, par delà tout effort de neutralité axiologique, le domaine d'étude des relations industrielles ne peut passer sous silence l'enjeu, réel et théorique, central des "relations" industrielles : qui maîtrise des institutions du monde du travail, qui autorise le contrôle de l'action collective dans ce monde?

Et au centre de cet enjeu, étant donné la logique dominante de l'employeur inhérente à la relation salariale, se pose la question éminemment institutionnelle de la position de contre-pouvoir dont pourraient disposer les salariés pour que le monde du travail salarié ne soit pas un lieu de domination abusive. Ainsi, la problématique du raisonnable (reasonabless) en matière de relations industrielles ne relève pas, comme d'aucuns le pensent, d'une dérive normative liée à des problèmes de "justice". Dans le domaine des relations salariales, comme pour tout ce qui touche au lien social, seules les institutions raisonnables sont vivables, seules sont soutenables et durables celles qui offrent à chaque salarié un avenir envisageable - une futurité significative commune socialement et individuellement acceptable - autre que la pure et simple perspective de domination de l'arbitraire patronal fondé sur la profitabilité. C'est dans cette optique du raisonnable, comme nécessité vitale des institutions salariales, que Commons aborde nécessairement normativement le champ des relations industrielles.

En effet, l'institution la plus raisonnable des relations industrielles pour une société en devenir (going concern) dans le monde du travail, c'est-à-dire pour une unité d'embauche de travail salarié par un employeur, est, selon Commons, celle où le processus de dé-re-structuration de la futurité significative commune est le plus raisonnable, c'est-à-dire celle offrant un processus permettant à la fois, d'une part, sous l'angle procédural, le meilleur mode d'autorisation des nouvelles solutions-sélections de règles opérantes de conduite, c'est-à-dire concrètement plutôt négociées ou acceptées comme légitimes que autoritaires et imposées par le pouvoir discrétionnaire patronal ou technocratique ${ }^{16}$, d'autre part, sous l'angle substantiel, la meilleure autorisation que puissent envisager les salariés étant données les autorisations déjà obtenues dans d'autres unités d'embauches, c'est-à-dire concrètement par exemple l'ajustement sur les meilleures clauses de convention collective en

\footnotetext{
16 C'est en rapport à cette question procédurale que Commons propose le paradigme de la common law comme forme historique de procédure d'autorisation des règles opérantes de conduite ayant certaines caractéristiques du raisonnable. Cependant, ce paradigme n'est pas pour Commons, et contrairement à ce que présentent nombreux de ses commentateurs, un modèle normatif prescriptif. En particulier, dans le domaine des relations industrielles, Commons est en faveur de la procédure de la négociation collective et, dès lors, la common law n'est que la procédure de recours ultime en cas de conflit irréductible (médiation arbitrage, etc.) entre les partenaires sociaux.
} 
vigueur ailleurs ou encore sur les meilleures pratiques connues dans le monde général du travail.

Pour que ces conditions soient remplies, Commons, et à sa suite l'École du Wisconsin en relations industrielles et sa lignée jusqu'à nos jours en Amérique du Nord (Kaufman 1993), recommande une présence syndicale légale et relativement puissante, selon un régime dont la concrétisation législative fut inaugurée par le Wagner Act de 1935 et qui est encore de nos jours le cadre institutionnel originel du système de relations industrielles en Amérique du Nord. Cette présence syndicale s'avère nécessaire, non pas seulement en vertu d'un principe formel de justice sociale - la démocratie industrielle et le droit à une pleine citoyenneté dans la société salariale -, mais aussi, et peut-être surtout d'un point de vue réaliste, pour rééquilibrer les forces en conflit de telle sorte que la futurité significative commune offre un univers vivable pour les salariés dans le monde du travail. Sans projet de vie au travail soutenable et durable pour les salariés, la futurité signifiante commune du monde du travail risquerait de devenir une "vision d'enfer" pour les salariés (pauvreté, précarité, insécurité, souffrance, anxiété, etc.), ce qui serait aussi contraire aux intérêts bien compris du patronat. En somme, pour Commons, la présence syndicale est nécessaire pour réduire la trop forte dissymétrie des forces d'autorisation en faveur des employeurs, pour que la puissance de l'autorité patronale quant à la détermination de ce qui sera autorisé les éléments de la futurité significative commune, c'est-à-dire les diverses règles opérantes de conduite prescrite ou permise de l'action collective contrôlante - ne soit pas trop fondée sur la coercition, la domination ou la subordination abusives. En somme, grâce au contre poids syndical, il s'agit de s'assurer que la "contrainte", dont nous avons vu plus haut qu'elle est un aspect incontournable de la relation salariale pour que puissent travailler "en concert" des acteurs sous un régime salarial, ne soit pas trop fondée sur le pouvoir de prédation et de subordination de l'employeur, c'est-à-dire que la futurité significative commune des salariés ne soit pas trop construite sous l'égide de la soumission à l'autorité arbitraire "légitime" patronale.

Sur la base de cette grille générale d'analyse des relations industrielles, l'approche de Commons peut dès lors se décliner analytiquement selon ce qu'il appréhende comme les divers types de transactions, c'est-à-dire les divers ensembles de règles opérantes de conduites constitutives de l'action collective, ou encore les divers espaces de la futurité significative commune, qui correspondent aux diverses facettes de la réalité institutionnelle du monde du travail. 


\section{2 - Les transactions salariales}

Commons propose de déployer l'analyse économique institutionnaliste à partir de l'unité analytique de base qu'est la transaction (Commons 1934a, 4). Sous l'angle ici privilégié dans l'approche commonsienne, à savoir la futurité significative commune, et en prenant toujours comme objet d'analyse la relation salariale, la transaction est l'élément structurel, réel et conceptuel, du troisième monde "pragmatique" permettant aux acteurs individuels, salariés et employeurs, de se projeter (futurité) en connaissance (significative) de cause (causalité institutionnelle) et en interaction (trans-action) plus ou moins contrôlée par l'action collective dans un à-venir en commun plus ou moins négocié ou concerté et plus ou moins sécuritaire pour chacun. L'enjeu et le contenu de la transaction sont donc, de ce point de vue, ce que sera (devra/pourra/pourrait être) la réalité avenir de la relation salariale pour les salariés et l'employeur. La dimension de temporalité (timeless) de la transaction en termes d'orientation/signification à-venir est tout à fait cruciale car sa prise en considération permet de séquencer, dans la réalité et dans l'analyse, le processus transactionnel complet de la relation salariale en trois moments successifs que Commons qualifie respectivement de négociationnel, transactionnel et administratif.

Par ailleurs, la relation salariale, comme toute autre relation économique, nécessite d'être étudiée en en différenciant trois facettes qui correspondent, dans la réalité et dans l'analyse, à trois types de transaction que Commons qualifie respectivement de transaction de marchandage (bargaining), transaction de direction (managerial) et transaction de répartition (rationing). Chacun de ces types de transaction, d'une part, relève respectivement d'un des trois principes constitutifs de la totalité de la réalité économique (échange, production, répartition), à savoir les principes de rareté (scarcity), d'efficacité (efficiency) et de souveraineté (sovereignty), d'autre part, assigne aux transacteurs des positions soit d'égalité (equality) soit d'inégalité de supérieur à inférieur, et ceci selon les moments successifs de la relation salariale.

En croisant ces deux conceptualisations, c'est-à-dire selon le processus temporalisé en trois phases et selon la typologie des transactions, on obtient la grille d'analyse commonsienne résumée dans le tableau I. Dans celle-ci, chacune des cases est, de plus, l'objet d'une conceptualisation commonsienne spécifique ainsi que le lieu d'analyse des conduites (position, situation) individuelles sous contrôle de l'action collective. Ainsi présentée, cette grille d'analyse commonsienne, jointe aux autres conceptualisations commonsiennes déjà évoquées et celles présentées dans le tableau II, permettent dès lors de caractériser la forme et le contenu de la formule (formula) de transaction spécifique à la dimension de la relation salariale étudiée. 
Cette formule est donc à la fois, d'une part, une formule idéaltypique, au sens weberien d'un outil analytique de compréhension de la réalité, c'est-à-dire selon Commons une configuration mentale (mental configuration) et une unité d'investigation (unit of investigation) qui n'est pas une copie de la réalité mais un moyen intellectuel de la comprendre (1934a, 59), d'autre part, une formule réel-typique, c'est-à-dire saisissant la réalité observable dans son contenu synthétique selon la méthode de 1'“étude de cas", méthodologie empirique dont Commons fut l'un des grands initiateurs en matière de relations industrielles et qui a concouru pour une bonne part à sa notoriété scientifique chez les praticiens de ce domaine du fait de son opérationnalité.

TABLEAU I

\begin{tabular}{|c|c|c|c|c|c|}
\hline Transaction & Principe & Relation & Négociationnelle & Transactionnelle & Administrative \\
\hline Marchandage & $\begin{array}{c}\text { Rareté } \\
\text { (ordre) } \\
\text { (propriété) }\end{array}$ & Egalité & $\begin{array}{c}\text { Persuasion/ } \\
\text { Coercition }\end{array}$ & $\begin{array}{c}\text { Dettes de } \\
\text { Performance/ } \\
\text { Paiement }\end{array}$ & Prix/Quantité \\
\hline Direction & $\begin{array}{c}\text { Efficacité } \\
\text { (coopération) } \\
\text { (efficacité) }\end{array}$ & $\begin{array}{c}\text { Supérieur/ } \\
\text { Inférieur }\end{array}$ & $\begin{array}{c}\text { Commande/ } \\
\text { Obéissance }\end{array}$ & Production & Intrant/Extrant \\
\hline Répartition & $\begin{array}{c}\text { Souveraineté } \\
\text { (citoyenneté) } \\
\text { (gouvernance) }\end{array}$ & $\begin{array}{c}\text { Supérieur/ } \\
\text { Inférieur }\end{array}$ & $\begin{array}{c}\text { Argumentation } \\
\text { Plaidoirie }\end{array}$ & (re)Distribution & $\begin{array}{c}\text { Charges/ } \\
\text { Bénéfices }\end{array}$ \\
\hline
\end{tabular}

TABLEAU II

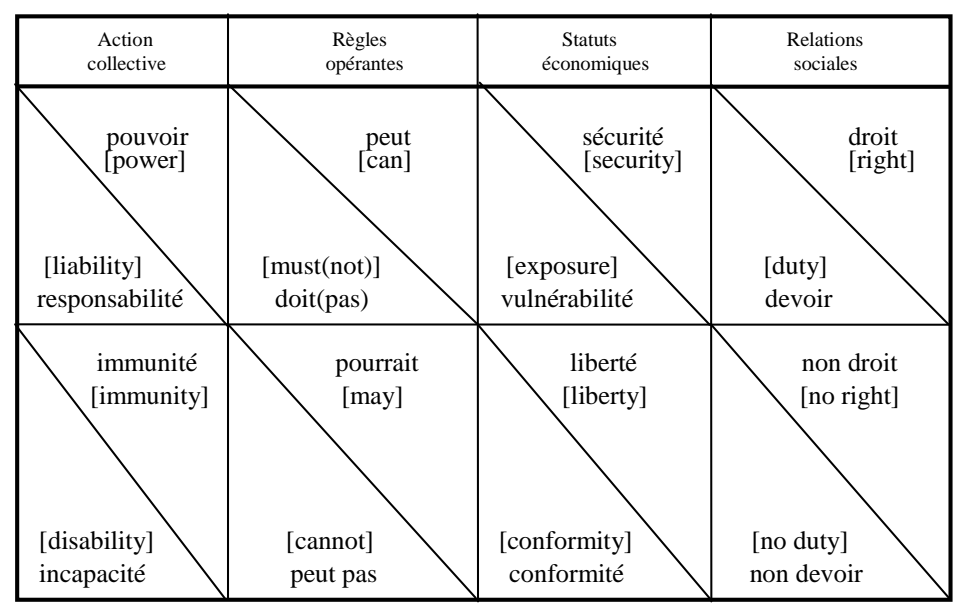

Source : Tableau recomposé à partir de celui de J. R. Commons (1934a) 
C'est en mobilisant cette grille d'analyse commonsienne, et en la complétant par d'autres apports conceptuels de l'analyse commonsienne, qu'il est maintenant possible de présenter les divers éléments séparés, mais devant être compris chacun comme une pertinence de l'analyse commonsienne d'ensemble, de l'institution de la relation salariale, champ d'étude des relations industrielles.

\section{1 - La transaction de marchandage salarial}

De façon générale, selon Commons, il n'existe pas d'harmonie naturelle préétablie dans le monde des relations économiques marchandes. Un ordre fondé sur un lien social institué est requis pour que puisse se déployer l'activité humaine dans ce monde. Cet ordre est assuré par l'existence d'une construction sociale, d'une institution, contrôlant le conflit permanent suscité par la rareté, c'està-dire par le fait que pour chacun les besoins excèdent les disponibilités en biens et services. Cette institution qui institue la relation marchande, c'est-à-dire qui est le préalable qui rend cette dernière socialement possible, est la propriété. Précisément en accord avec la conception de la futurité significative commune comme expression condensée du fondement temporalisé de l'institution chez Commons, la propriété est définie par ce dernier comme un droit légal sur l'usage futur d'un bien ou service, c'est-à-dire ce sur quoi les transacteurs s'entendent (signification commune), et que le droit garantit, quant à quel transacteur aura (futurité) l'usage futur d'un bien ou service.

Ce fondement institutionnel, spécifique au type de transaction qu'est la transaction de marchandage, s'applique aussi à la transaction de marchandage salarial. L'objet de cette dernière concerne donc la question de la "propriété" du service salarial, c'està-dire la question du droit de cession quant à l'usage futur de la force de travail du salarié. Cette conception commonsienne rappelle ainsi que la relation salariale est d'abord un "louage" : la cession d'un droit d'usage de quelque chose, le service laborieux du salarié, pour un certain temps contre une rémunération de type loyer, le salaire. Ce droit d'usage est cédé par celui qui en détenait antérieurement la propriété, le candidat salarié, au profit de celui qui le détiendra à l'avenir, l'employeur putatif. Ce premier aspect de l'institution de la relation salariale permet d'emblée à Commons d'établir la connexion, analytique et réelle, d'une part, entre l'économie, la relation marchande salariale, et le droit, les aspects juridiques (cession, usage, usufruit) de cette relation bien particulière de louage, d'autre part, entre l'économie et l'éthique, ce "louage" est celui d'une personne et non d'une chose. Ainsi, dès ce premier fondement de la transaction de marchandage salarial, l'institution de la relation salariale ne peut être comprise d'un point 
de vue purement économiciste ou, pour reprendre la formulation de l'économie politique orthodoxe, du point de vue de l'économie "pure", c'est-à-dire de celui de l'autre troisième monde fictif que serait l'économie pure de "marché".

D'ailleurs et de façon plus générale, souligne Commons, les trans-acteurs dans toute transaction de marchandage doivent être dans une relation, instituée en droit, d'égalité. Cette égalité de droit n'est effective, en réalité, que si tous les éléments structuraux de la formule typique de la transaction de marchandage sont respectés, et ceci est aussi vrai pour la transaction de marchandage salarial. En effet, dans ce dernier cas, il faut qu'il y ait : au moins deux transacteurs salariés candidats, c'est-à-dire au moins deux candidats loueurs d'un même type de service laborieux; au moins deux transacteurs employeurs utilisateurs putatifs de celui-ci; et une autorité autorisée, en général les instances juridiques légalement compétentes, qui fasse respecter l'égalité de droit entre ces deux catégories de trans-acteurs et donc autorise cette transaction.

La pluralité, au moins deux pour chaque catégorie, des transacteurs est une condition nécessaire (et non suffisante) d'égale opportunité entre eux, d'égal choix alternatif entre plusieurs opportunités, d'emplois pour le salarié et de candidats pour l'employeurs, en somme d'égale situation et position de marchandage dans la transaction. Si tel n'est pas le cas, si par exemple le salarié n'a pas de choix alternatif face à une seule offre d'emploi, s'il n'a donc pas d'autres opportunités d'emplois que cette offre et qu'il ne peut pas attendre l'apparition d'une autre offre, alors que l'employeur dispose déjà de plusieurs candidatures et peut attendre pour embaucher, dans ces conditions d'inégale opportunité, le pouvoir de marchandage (bargaining power) de l'employeur est très nettement supérieur à celui du salarié. Dans ce cas le plus fréquent de dissymétrie, la futurité significative commune du salarié candidat est très contrôlée et contraignante. Ce salarié doit "forcément" accepter l'emploi, il est fortement incité à la performance (performence), accepter l'emploi, et son action est donc contrainte (restraint), il n'a pas d'autre choix que d'accepter cet emploi. A l'inverse, dans la même situation mais cette fois du côté de la position de l'employeur putatif, la futurité significative commune de ce dernier est moins contrôlée et plus ouverte. Cet employeur a le choix entre la performance (performence), embaucher ce salarié, le réfrènement (forbearance), l'embaucher partiellement, ou l'évitement (avoidance), ne pas l'embaucher. Son action est donc ainsi libérée (liberation), l'action collective l'appuie dans le choix de son action, et cette dernière peut même être en expansion (expansion), il a une ouverture d'action alternative, certains salariés candidats peuvent être recrutés sur d'autres postes. 
Cette question des pouvoirs de marchandage respectifs des trans-acteurs est tout à fait centrale dans la première phase de la transaction de marchandage salarial, celle que Commons qualifie de négociationnelle (negociational). En effet, selon le degré de dissymétrie des pouvoirs de marchandage respectifs des transacteurs, la psychologie négociationnelle (negotiational psychology) respective de ces derniers sera de qualité différente. Ceci aura pour conséquence soit une situation transactionnelle de persuasion (persuasion), c'està-dire de relative égalité réelle entre les transacteurs, soit de coercition (coercion), c'est-à-dire de pouvoir de marchandage abusif de l'un des transacteurs sur l'autre. Dans la situation réel-typique la plus courante, celle où le contenu idéal-typique de la formule de transaction de marchandage salarial est structuré de telle sorte que le salarié candidat est soumis à la coercition de l'employeur putatif, la futurité significative commune dans laquelle s'inscrit ce salarié, la façon dont est instituée pour lui l'action collective dans ce premier moment négociationnel de la transaction de marchandage salarial, est caractérisée par l'incapacité (disability) face à l'immunité (immunity) de l'employeur putatif. Cette situation trans-actionnelle dissymétrique se traduit par une position pour le salarié candidat dans laquelle le contrôle des règles opérantes de l'action collective ainsi instituée l'incite (inducement) fortement à adopter une conduite spécifique socialement construite : celle de devoir (duty), il doit (must) prendre cet emploi ; celle de non droit (no right), il n'a pas le droit à l'égalité des chances et à l'opportunité d'obtenir un autre emploi; celle où il ne doit pas (must not) refuser cet emploi. Dans ces conditions, ce salarié candidat est institué selon un statut (statut) économique de vulnérabilité (exposure) et réciproquement l'employeur putatif dispose d'un statut de sécurité (security), et cette situation transactionnelle met ce salarié en position précaire de conformité (conformity) alors que, réciproquement, l'employeur possède un statut de liberté (liberty). Cette situation statutaire contraint le salarié candidat à une position de non choix et donc à se conformer à ce que lui prescrit l'action collective ainsi instituée. Avec un tel statut, un statut étant selon Commons caractérisé par " une attente de règles opérantes suivant laquelle l'individu ajuste son comportement présent " (1934a, 412), et dans notre cas l'attente contrôlant le salarié candidat s'inscrit dans une futurité significative commune ne lui proposant comme projet (purpose) possible que d'accepter l'emploi, ce salarié ne possède alors qu'un seul registre d'hypothèses habituelles (habitual assumptions) guidant son action individuelle. Ces hypothèses habituelles, celles-ci étant en général selon Commons " les attentes de toutes les parties concernant les futures conséquences économiques de leurs transactions présentes " (1934a, 84), ne lui offrent qu'une possibilité d'action, qu'un pouvoir 
d'action (power of action), au demeurant bien restreint et contraint : accepter l'emploi.

On aura compris que dans cette analyse commonsienne de la première phase négociationnelle de la transaction de marchandage salarial, la question du choix "libre" du salarié candidat, telle que nous la présente la théorie économique orthodoxe, n'a pas de grande pertinence. Étant donné la variété des situations négociationnelles dans lesquelles sont respectivement les salariés candidats et des employeurs putatifs dans les transactions de marchandage salarial, les questions les plus pertinentes pour l'analyse institutionnaliste commonsienne sont de deux ordres. D'une part, il s'agit de caractériser analytiquement la structure idéal-typique de la formule de transaction de marchandage salarial dans sa phase négociationnelle et d'en déterminer le contenu concret réel-typique quant aux positions et situations instituées des trans-acteurs. En d'autres termes, il s'agit de savoir ce qu'offre respectivement la futurité significative commune instituée aux trans-acteurs, ce que sont leurs statuts économiques tels que déterminés par la structure de l'action collective, c'est-à-dire par les diverses règles opérantes qui la composent, et quels sont donc les possibilités et pouvoirs d'action respectifs des transacteurs. D'autre part, en conséquence et normativement, il s'agit de déterminer quelles sont les solutions institutionnelles envisageables raisonnablement pour réduire la dissymétrie des pouvoirs de marchandage dans la phase négociationnelle de la transaction de marchandage salarial.

C'est l'examen de cette seconde question qui conduit Commons à proposer, comme solution raisonnable de "rééquilibrage" des pouvoirs de marchande, la présence légale d'un syndicat suffisamment puissant pour concourir, par la négociation, la concertation, le partenariat, etc., à instituer une futurité significative commune, c'est-à-dire une formule du moment négociationnel de la transaction de marchandage salarial, réduisant autant que possible le statut de vulnérabilité des salariés candidats au louage de leurs services laborieux. L'existence d'une coercition abusive pesant sur ces salariés risque de réactiver le conflit latent et de remettre en cause l'ordre social institué par la propriété, celui de l'institution du droit légal d'usage futur du "service laborieux" du salarié par l'employeur. Ce risque que court l'institution du salariat, faute d'être éliminé par l'institution du (contre)pouvoir syndical, devra alors être éliminé par l'intervention d'une autorité autorisée à fournir des solutions institutionnelles. Que cette autorité, selon les caractéristiques soulignés plus haut, soit le législateur, une instance judiciaire compétente, une instance professionnelles ou toute autre reconnue dans la société civile, une instance ou personnalité autorisée au sein du going concern utilisateur de salariés, etc., dans tous les cas, une solution institutionnelle liant économie, droit et 
éthique ne peut pas ne pas être recherchée et trouvée. S'il y a bien une transaction de marchandage dont le fondement institutionnel est socialement nécessaire dès le premier moment de la phase négociationnelle, c'est-à-dire où est requise au préalable l'existence d'un ordre propriétaire (proprietary order) quant au droit légal de l'usage futur et pour laquelle à la futurité significative commune des transacteurs nécessite d'être raisonnablement sécurisée, en particulier concernant le statut des certains transacteurs, c'est manifestement la transaction de marchandage salarial ${ }^{17}$.

Le second moment de la transaction de marchandage est en général celui de la phase que Commons qualifie de transactionnelle. En effet, une fois les transacteurs campés par leurs situations, dans la phase négociationnelle, sur leurs positions et statuts d'acteurs respectifs, il s'agit maintenant pour eux d'arriver à concrétiser des engagements pour l'action future (commitments for futur action). Ces engagements peuvent prendre multiples formes - accords, contrats, obligations réciproques, règles d'action communes, conjointes, etc.selon l'objet de la transaction de marchandage et quant aux droits légaux d'usage futur des divers biens ou services. En somme, il s'agit de formaliser des engagements quant à la futurité significative commune : quels seront les élément constitutifs de l'action collective à-venir ? qu'est ce que respectivement les transacteurs devront(pas), pourront(pas) ou pourraient(pas) faire en concert ? Dans sa dimension la plus générale, ces engagements portent sur deux éléments respectifs de contrepartie : d'une part, une dette de livraison (pour un bien) ou de performance (pour un service) à terme à laquelle s'engage le vendeur, d'autre part, une dette de paiement à terme à laquelle s'engage l'acheteur. Ces engagements sous forme de double dette s'appliquent aussi à la phase transactionnelle de la transaction de marchandage salarial.

Le salarié candidat qui accepte l'emploi s'engage à performer pendant son activité salariée, il reconnaît ainsi à son futur employeur une dette de performance à terme. Cette reconnaissance de dette de performance peut prendre un aspect formel en tant que libellé dans un contrat de travail mais peut aussi, pour les aspects difficilement formulables ou dont les descriptions seraient interminables, consister en des énoncés verbaux ou des considérations implicites quant à la dite performance à livrer dans le futur. Ce caractère bien évidemment incomplet et parfois vague de la dette de performance du futur salarié pose incontestablement un problème d'interprétation dont les effets s'exerceront, comme nous le verrons plus bas, lorsqu'il sera question de la transaction de direction. De façon générale, au stade de l'engagement de

17 Cette problématique commonsienne est l'objet de l'article de S. Morel dans ce numéro. 
performance de la part du salarié, deux caractéristiques institutionnelles feront que cet engagement est plus ou moins ouvert ou fermé. La première caractéristique relève du statut économique institué dans laquelle se trouvait le salarié candidat au moment de la phase négociationnelle de la transaction de marchandage salarial. Plus (moins) ce statut était celui de vulnérabilité (sécurité), plus (moins) l'engagement risquera d'être ouvert (fermé) et en faveur de l'interprétation future de l'employeur. La seconde caractéristique concerne l'état présent et surtout futur des éléments structurant l'action collective de l'activité salariale dans laquelle le salarié promet de s'insérer. Plus (moins) cette action collective sera organisée, c'està-dire sera régie par des règles opérantes de conduite formelles structurant la futurité significative commune de l'activité du futur salarié, ou sera inorganisée mais régie selon des principes d'autonomie salariale, plus (moins) l'engagement sera fermé (ouvert) et en faveur de l'interprétation future du salarié.

Dans une situation réel-typique, souvent la plus fréquente, où le contenu idéal-typique de la formule de transaction de marchandage salarial est structuré selon un degré d'ouverture fortement favorable à l'interprétation de l'employeur quant aux caractéristiques de l'engagement de performance salariale, se pose le problème institutionnel de l'arbitraire patronal. Lorsque cet arbitraire risque d'être potentiellement abusif et coercitif dans l'exécution futur de l'engagement de performance salariale, la question de l'ordre, dont nous avons vu qu'il est un préalable nécessaire à toute transaction de marchandage, est à nouveau posée car un tel engagement serait trop difficilement vivable pour le futur salarié, lui imposerait une futurité significative commune en termes de futures règles opérantes de conduite à suivre trop coercitives. Le conflit ouvert qui pourrait s'ensuivre remettrait en cause l'ordre institué, c'est-à-dire la légitimité du droit légal d'un usage futur abusif des "services" du salarié. Le conflit sur le caractère abusif ou non d'un tel type d'engagement du salarié nécessite d'être tranché par une autorité autorisée, en général les instances juridiques reconnues compétentes en ce domaine, qui aura à donner l'autorisation ou non, à légitimer ou non le contenu d'un tel engagement. A nouveau, dans cette approche commonsienne, la connexion économie/droit/éthique s'avère incontournable. Par delà tout argumentaire strictement économiciste fondé sur le constat de la (non)rareté, c'est-à-dire sur l'abondance et la disponibilité de candidats salariés contraints à devoir accepter de tels engagements abusifs du fait de leur faible pouvoir de marchandage dans la phase négociationnelle de la transaction de marchandage salarial, l'institution de la relation salariale, pour être raisonnablement légitime, requiert une certaine conformité au droit et à l'éthique appliquée à la condition salariale. Il ne s'agit pas uniquement d'une question de "normativité" qui serait 
"externe" à la question "purement" économique de la relation salariale, il s'agit d'une question de nécessité préalable à l'institution du salariat. Une telle institution, et la relation salariale ne peut être possible et comprise autrement que comme une institution, ne peut exister que si elle est autorisée par le droit et l'éthique reconnus dans la société salariale et, lorsque le conflit est ouvert, sanctionnée par les instances d'autorisation (le législatif, le judiciaire, etc.).

Commons ne remet pas en cause la légitimité et même la nécessité ultime des autorités législative et judiciaire, bien qu'il préfère d'autres formes quasi-para-publiques d'administration des relations industrielles (commission des relations industrielles, instances de concertations, etc.) auxquelles d'ailleurs il a fortement contribué à définir l'architecture et l'opérationnalité dans le premier vingtième siècle états-unien (1934b). Toutefois, Commons considère qu'une présence syndicale légale et suffisamment puissante est une garantie institutionnelle nécessaire pour défendre collectivement les salariés contre les abus coercitifs issus de l'excessif pouvoir de marchandage des employeurs et se concrétisant par des engagements de performance salariale déraisonnables. La convention collective avec ses clauses "normatives" définissant les "tâches et charges de travail", et au moyen de laquelle les salariés peuvent négocier collectivement des engagements de performance relativement raisonnables, est, d'une part, pour les futurs salariés la garantie d'une futurité significative commune relativement acceptable quant aux "conditions de travail", d'autre part, pour les futurs employeurs une garantie d'autorisations quant à l'usage futur des services salariés, c'est-à-dire une futurité significative commune relativement organisées pour les besoins de 1"exploitation" salariale. En somme, la convention collective sécurise relativement la futurité significative commune salariés/employeurs, détermine une part de l'action collective contrôlant les actions individuelles, c'est-à-dire des règles opérantes de conduites organisées en vue de l'action concertée nécessaire à l'activité productive. Ces considérations sur la nécessaire institutionnalisation, de préférence formelle et organisée par la convention collective, de la relation salariale dans la phase transactionnelle de la transaction de marchandage salarial se retrouvent aussi, pour Commons, lorsqu'il s'agit de la contrepartie monétaire à l'engagement de performance salariale.

En effet, en contrepartie de l'engagement/dette de performance salariale, l'employeur s'engage à rémunérer le futur salarié au terme de sa performance, il lui reconnaît une dette de paiement, un salaire payable à terme. Cette question de la détermination du salaire est abordée par Commons d'un double point de vue complémentaire : celui du montant du salaire et celui de la dimension monétaire du salaire. 
Concernant la question du montant du salaire, une bonne part de l'analyse commonsienne ayant pour objet l'engagement de performance salariale est reprise en l'appliquant à la question de la concrétisation de la dissymétrie des pouvoirs de marchandage en matière de salaire. Dans la situation la plus fréquente où le salarié candidat subit le pouvoir de coercition abusif de l'employeur putatif, le montant du salaire promis dépendra de la prégnance du statut de vulnérabilité (exposure) et de conformité (conformity) que subit le salarié candidat (incitation contraignante à devoir performer dans l'incapacité à ne pouvoir refuser de façon responsable le salaire offert faute d'alternative) face à la liberté (liberty) et la sécurité (security) de l'employeur putatif (choix de l'action d'offrir un certain montant de salaire grâce au pouvoir, à l'immunité et au droit qui lui permettent d'autres opportunités d'embauche). Ainsi, dans une telle situation, la futurité significative commune qui se présente au salarié candidat, à savoir un salaire futur qui tend vers sa limite déterminé par la (non)rareté de candidats concurrents (limite qui d'ailleurs selon cette logique purement économiciste peut tendre vers zéro en situation d'abondance de main-d'œuvre disponible), peut difficilement être vivable pour lui et l'inciter à remettre en cause l'institution du salariat telle qu'elle se présente à lui : une action collective faiblement organisée qui le contrôle fortement dans le sens de la coercition abusive de la part de l'employeur putatif. Dans ces conditions, ce qui a été présenté plus haut concernant l'institution raisonnable déterminant l'engagement de performance productive s'applique aussi ici. Un dispositif institutionnel (législatif, judiciaire, administratif) d'autorisation et surtout de négociation collective avec présence légale syndicale suffisamment puissante s'avère institutionnellement et raisonnablement nécessaire quant à la détermination des montants de salaires. Selon Commons, un monde de relations industrielles n'étant pas régi par ces institutions d'autorisation légale et de négociation collective pourraient certes exister, et il a pu existé dans des temps passés marqués par l'extrême précarité de la condition salariale, mais d'un point de vue réaliste, notamment quant à la connexion économie/droit/éthique, et institutionnaliste ce monde n'est pas raisonnable et/car contraire à l'institution du salariat comme futurité significative commune suffisamment vivable (soutenable et durable) pour les salariés.

Le fait que le salaire a une dimension monétaire est aussi tout à fait important pour l'analyse commonsienne ${ }^{18}$. En effet, deux aspects de cette dimension monétaire sont cruciaux pour comprendre pourquoi et comment la relation salariale est une institution économique. Le premier de cet aspect concerne le fait que l'engagement de l'employeur putatif s'exprime par une dette de

${ }^{18}$ Sur la théorie monétaire de Commons, voir C. J. Whalen (1993) et J. Maucourant (1993, 2001). 
paiement. A la différence de la dette de livraison sous forme d'engagement de performance de la part du salarié candidat qui entraîne de nombreux problèmes d'identification, de qualification et d'inscription dans la temporalité ainsi que de formulation, de formalisation contractuelle et d'interprétation entre les transacteurs de la relation salariale, la dette de paiement libellée en monnaie est triplement précise. Il s'agit d'une quantité de monnaie légale payable à un terme défini dans le temps chronologique.

Le salaire monétaire est une quantité chiffrable sans équivoque et en ce sens offre une mesure objective, socialement reconnue et observable, quant au contenu de la futurité significative commune pour les transacteurs de la relation salariale. Ces derniers s'entendent, ce type d'entente étant le principe même de tout lien social institué, sur la convention numérique monétaire. Il s'agit donc bien, pour Commons, d'une institution de quantité dont la portée, comme fait social institué, est cruciale pour comprendre l'une des caractéristiques essentielles de l'institution de l'économie : non un fait de nature objective extérieure - le premier monde cartésien -, ni un fait de nature subjective intérieure - le seconde monde cartésien , mais un fait institué constitutif, comme élément structurant, du troisième monde institutionnel, mis en lumière par la philosophie pragmatiste, de la futurité significative commune, c'est-à-dire une grandeur significative sur laquelle chacun s'accorde (croyance et habitude communes) pour projeter (futurité) son action "économique". Dans ces conditions, la dette de paiement que constitue le salaire est exemplaire de l'institution économique : cette dette objective, selon une grandeur significative commune, une projection dans le monde économique. Le temps chronologique de la relation salariale trouve ainsi sa traduction économique instituee dans la temporalité (celles des "période" et "durée" salariales) et la grandeur (monétaire) spécifiquement économiques du salaire payable à terme. Cette empreinte fortement instituée de la relation salariale $\mathrm{du}$ fait de son inscription dans une logique proprement économique de dette monétaire à terme explique sans doute l'âpreté du conflit entre salarié candidat et employeur putatif sur la question du salaire. Ce qui se joue dans la détermination du salaire, outre bien entendu ce que celui-ci impliquera comme pouvoir d'achat pour le salarié et comme profit pour l'employeur, c'est l'avenir de ces transacteurs dans le monde objectivé, car institué sans équivoque, de la seule grandeur économique socialement reconnue/partagée : la grandeur monétaire et sa forme instituée la plus pure qu'est la dette monétaire.

Le second aspect de la dimension monétaire du salaire comme dette de paiement qui permet de comprendre pourquoi et comment la relation salariale est une institution économique est que cette dernière est intimement liée au fait que la monnaie est aussi, et 
sans doute la première d'entre elles, une institution économique. Nous avons vu comment Commons met en évidence le fait que la monnaie est ce qui permet l'institution de la seule grandeur spécifiquement économique et, en conséquence, donne à la dimension monétaire du salaire dans la relation salariale l'une des caractéristiques les plus prégnantes de l'institutionnalisation de cette dernière. Il en découle donc que, pour une bonne part, la solidité institutionnelle de la relation salariale dépend de la solidité de l'institution monétaire. Dans le cas où cette dernière serait déstabilisée, les effets négatifs sur la solidité de l'institution de la relation salariale seraient inévitables. Si l'institution monétaire perd de sa crédibilité sociale, les effets sur l'institution de la relation salariale seraient, peut-être plus que dans tout autre domaine de l'activité économique, de déstructurer et d'insécuriser la futurité significative commune des transacteurs. Si le montant monétaire du salaire-dette n'est plus une grandeur économique envers laquelle la confiance règne, alors l'avenir des transacteurs de la relation salariale n'est plus envisageable selon un certain degré raisonnablement nécessaire de certitude, leur futurité significative commune devient hautement insécure. $\mathrm{Si}$, de plus, la dégradation institutionnelle de la monnaie s'accompagne d'une dissymétrie d'effets respectifs sur les salariés et les employeurs et en faveur de ces derniers, dans le cas par exemple où le pouvoir d'achat des salaires nominaux serait plus affecté que celui des profits nominaux, alors les conflits lors de la phase transactionnelle de la transaction de marchandage salarial risquent fort de mettre à mal l'institution anciennement établie de la relation salariale. Ce poserait alors à nouveau le problème de l'ordre institutionnel requis pour que puisse raisonnablement s'instituer la relation salariale. Les autorités responsables de l'institution monétaire seraient dès lors socialement convoquées à en rétablir la crédibilité sociale et à éliminer les effets inégalitaires de l'instabilité monétaire entre salariés et employeurs. C'est dans cette optique de protection de l'institution monétaire et de stabilisation monétaire, en particulier pour garantir l'avenir du pouvoir d'achat salarial et donc la solidité institutionnelle de la relation salariale, que Commons s'est engagé, avec d'autres économistes de son temps et notamment avec I. Fisher, dans la lutte pour l'élaboration sociale d'une institution monétaire stable, en particulier grâce à l'action interventionniste de la Réserve Fédérale nouvellement créée.

Le troisième moment de la transaction de marchandage est celui que Commons qualifie d'administratif. Ce qui n'était encore que négociation (premier moment) et engagements transactionnels (second moment), c'est-à-dire construction et structuration de la futurité significative commune, l'élaboration d'un troisième monde 
institutionnel putatif, est maintenant concrétisation et réalisation effectives dans le présent. Le droit d'usage légal futur et les engagements de double dette livraison-performance / paiement deviennent réalité présente : il s'agit maintenant d'administrer la futurité antérieure, de la faire entrer dans les faits, d'en faire la réalité économique avec ses prix - extinction/libération de dette de paiement - et ses quantités - extinction/libération de dette de livraison-performance-.

La théorie commonsienne des prix et des quantités, les uns et les autres ne se réalisant effectivement qu'au moment de la phase administrative de la transaction de marchandage, est donc construite sur la base méthodologique, issue de la philosophie pragmatiste, du principe de causalité institutionnelle: le processus de construction opérationnelle de la futurité significative commune étant arrivé à son terme (à l'issue des deux premières phases), cette futurité (cause) entraîne de par son administration (au sens de l'exécution d'une prescription) des conséquences (effets) économiques réelles qui se concrétisent dans les prix et les quantités. Ces derniers ne sont donc pas des faits indépendants du processus et de la temporalité de l'activité économique trans-actionnelle, ils ne sont pas des faits mécaniques d"équilibre" ou des faits "émergents" inintentionnels, ils sont l'expression concrète du principe de réalité économique institutionnelle fondée sur la futurité significative commune mise en œuvre, opérationnalisée, réalisée, exécutée, etc. par les transacteurs. Et comme ces derniers, comme nous l'avons vu précédemment, n'ont pas les mêmes statuts économiques, ne sont pas dans les mêmes positions réciproques de pouvoir / responsabilité, immunité / incapacité, sécurité / vulnérabilité, droit / devoir, etc. selon les différentes situations de transaction (tableau II), les prix et les quantités qui concrétisent ces rapports dissymétriques de statuts sont aussi variés comme résultats, dans la phase administrative de la transaction de marchandage, qu'il existe de différences qualitatives entre les contenus concrets des formules de transaction.

Seule l'étude de cas, aidée des instruments d'analyse des formules idéal-typique et de leur contenu réel-typique, peut permettre de rendre compréhensible tel ou tel prix et telle ou telle quantité dans telle ou telle transaction de marchandage. Dans l'optique institutionnaliste de Commons, le processus de "formation" des prix et des quantités ne relève donc pas d'une logique physicaliste (premier monde) et/ou introspective (second monde), il concerne plutôt une logique de mise en oeuvre de la causalité institutionnelle selon une futurité significative commune, c'est-à-dire selon les projets respectifs sur lesquels les acteurs économiques s'entendent malgré leur dissymétrie de statut dans les transactions, se concrétisant dans la réalité économique. En somme, ce sont les 
caractéristiques spécifiques de l'action collective dans une transaction de marchandage particulière qui déterminent les prix et les quantités. Dans l'approche commonsienne, la "formation" des prix et des quantités résulte donc d'un processus transactionnel toujours spécifique quant aux contenus analytique idéal-typitique et concret réel-typique de la formule de chaque transaction de marchandage particulière.

L'analyse commonsienne générale de la troisième phase administrative s'applique aussi à la transaction de marchandage salarial. Dans ce cas, le prix de l'usage de la force de travail salariée, c'est-à-dire le salaire monétaire, se concrétise par le paiement de sa dette par l'employeur à l'issue de la période de travail. Le prix/salaire est donc une libération/extinction de dette de paiement. En contrepartie, la quantité de travail fourni à l'issue de cette même période correspond à une libération/extinction de dette de performance par le salarié en faveur de l'employeur. A ce dernier stade de la transaction de marchandage salarial, deux éléments peuvent donc poser des problèmes à l'institution salariale. L'un d'eux concerne le conflit qui peut toujours survenir entre salarié et employeur sur la quantité livrée de performance laborieuse. Bien qu'il s'agisse conceptuellement d'une quantité économique, au sens déjà évoqué de référence à une grandeur spécifique à l'institution économique, la mesure possible de cette quantité est beaucoup plus équivoque, prête à beaucoup plus de problèmes d'interprétation, que pour le cas des quantités monétaires. Nous aborderons plus en détails cette problématique commonsienne à propos de l'analyse de la transaction de direction salariale car cette problématique est centrale pour cette dernière.

L'autre élément qui peut prêter à conflit dans la phase administrative de la transaction de marchandage salarial est bien entendu la question du paiement de la dette salariale : le salaire versé est-il à la hauteur de la dette de paiement promis par l'employeur ? Deux sous questions peuvent être dissociées. La première est relative à l'adéquation entre la quantité de performance livrée par le salarié et le salaire effectivement payé par l'employeur et ceci en rapport avec ce qui avait été entendu lors de la phase transactionnelle. Si on laisse à nouveau de côté, pour la même raison que ci-dessus, le problème de l'évaluation de la quantité de labeur effectivement fournie par le salarié pour s'intéresser uniquement au problème de l'adéquation entre performance effective et salaire versé, la question ici est celle du respect des engagements. Et plus ces engagements auront été vagues et matières à interprétation, ou pour l'exprimer conceptuellement, plus le vacuum de la futurité significative commune en cette matière sera important, plus le conflit risque d'apparaitre et de fragiliser l'institution salariale. Dans une telle situation, seule une autorité, une instance judiciaire ou 
administrative de règlement ou d'arbitrage de grief, peut trancher le conflit et, de la sorte, maintenir la légitimité institutionnelle de la relation salariale. A nouveau, on retrouve ici la connexion économie/droit/éthique et la nécessité de sa prise en compte pour que le conflit soit réglé dans une optique consolidant l'institution salariale. Cependant, bien que Commons soit en faveur de telles instances d'autorisation et ait, comme nous l'avons déjà signalé fortement contribué à leur établissement institutionnel, sa préférence va à l'établissement d'institutions salariales avec présence syndicale légale et suffisamment puissante pour protéger les salariés a priori, dans les clauses de la convention collective au moment de la phase transactionnelle de la transaction de marchandage salarial, ou $a$ posteriori, grâce à des instances internes, avec représentation des salariés, de règlement de conflit interne au going concern salarial.

La seconde sous question évoquée plus haut concerne la stabilité, en termes de mesure réelle, de la quantité monétaire du salaire versé, c'est-à-dire la stabilité du pouvoir d'achat ou salaire réel versé par rapport au salaire nominal promis et versé. Cette question nous ramène à une problématique similaire à celle déjà rencontrée à propos de la dimension monétaire de la phase transactionnelle de la transaction de marchandage salarial mais cette fois, non plus d'un point de vue putatif de la futurité significative commune à établir quant au montant du salaire monétaire avenir, mais du point de vue de la différence entre l'engagement de dette de paiement salarial et la réalisation en quantité effective de salaire versé. La solution générale préconisée par Commons est la même que précédemment quant aux questions monétaires : la consolidation et la stabilisation d'institutions monétaires protégeant la légitimé monétaire et le pouvoir d'achat de la monnaie et donc des salaires nominaux. A défaut de telles institutions générales, Commons est en faveur d'institutions garantissant, notamment grâce à la présence syndicale, par voie de négociation, concertation, etc., le pouvoir d'achat des salaires. Ces questions monétaires, qui peuvent paraitre éloignées de l'analyse de la relation salariale, sont en fait très importantes pour Commons concernant cette analyse. Outre la question particulière du pouvoir d'achat salarial, Commons s'intéresse à l'institution monétaire car il considère qu'elle est un fondement crucial de l'institution salariale. La dette de paiement qu'est le salaire correspond à la forme la plus pure de l'institution monétaire : une dette, c'est-à-dire une projection dans la futurité, et une mesure, c'est-à-dire une quantification de cette futurité, sur la base desquelles il est possible de construire une futurité significative commune en économie, en somme sur lesquelles les transacteurs économiques, salariés et employeurs, peuvent communément bâtir leurs croyances chiffrables et régler leurs conduites habituelles. Ainsi, l'institution monétaire est une condition 
nécessaire de l'action collective en économie et en particulier pour la relation salariale.

En résumé, pour Commons, ce sont les trois moments successifs, négociationnel, transactionnel et administratif, qu'il faut considérer pour que ce premier type de transaction de la relation salariale, la transaction de marchandage salarial, puisse être analysé en totalité et pour que, in fine, soit rendus compréhensibles les prix, les salaires, et les quantités, les droits d'usage du service laborieux. L'étude commonsienne des deux autres types de transaction, de direction et de répartition, constitutifs de la relation salariale complète cette première analyse.

\section{2 - La transaction de direction salariale}

De façon générale, selon Commons, dans une société où l'activité productive est assurée dans des going concerns tels que des établissements de production, c'est-à-dire des regroupements humains de production "en concert", l'efficacité productive et la performance technique des travailleurs requièrent une coordination entre eux. Ceci ne peut se réaliser que si est institué un principe de coopération. Ce dernier est un principe général à tout régime de travail, y compris donc pour le régime salarial, car il est la condition nécessaire de l'efficacité de la lutte contre la rareté. Ainsi, alors que la question sociale de la rareté et l'institution de la propriété qui maintient l'ordre social face à cette rareté sont au fondement de la transaction de marchandage, la question technique de l'abondance, c'est-à-dire de la réduction de la rareté, et en conséquence le principe de coopération requis entre travailleurs pour assurer l'efficacité productive, sont au fondement de la transaction de direction (managerial transaction), c'est-à-dire du type spécifique de transaction qui permet un ordre productif efficace.

L'approche institutionnaliste commonsienne dissocie donc analytiquement, grâce à ces deux types d'unité d'analyse que sont respectivement les transactions de marchandage et de direction, ce qui relève classiquement dans la théorie économique, d'une part, de la division sociale du travail et donc des questions de rareté / propriété, ce que les économistes orthodoxes appellent les phénomènes de "marché", d'autre part, de la division technique du travail et donc des questions d'abondance productive / coopération technique efficace, ce que les sociologues et certains économistes appellent les phénomènes d"organisation". Cependant, cette dissociation analytique ne signifie pas dans l'approche commonsienne, à la différence de l'approche économique orthodoxe ou de celle de la spécialisation disciplinaire entre "économistes" s'intéressant au "marché" et "sociologues" s'intéressant à l'organisation", qu'il y aurait deux réalités économiques exclusives 
l'une de l'autre, une sorte d'alternative entre une économie de "marché" et une économie "organisée". L'approche commonsienne, et c'est ce qui contribue aussi à sa puissance analytique, décline deux facettes d'une même réalité économique régie conjointement par deux principes d'économicité, celui de rareté/propriété/marchandage (bargaining) et celui d'efficacité productive / coopération technique/direction (management). Ces deux principes respectifs s'appliquent spécifiquement dans un même going concern économique selon qu'il s'agit soit, dans les transactions de marchandage, de faire des affaires (going business), soit dans les transactions de direction, de faire de la production (going plant). De plus, à la différence de la dichotomie veblenienne qui oppose deux logiques économiques antagoniques, celle socialement négative (prédation, rivalité pécuniaire, etc.) des affaires (business) et celle positive (technique instrumentale, travail utile, etc.) de l'industrie (industry), l'approche commonsienne articule, sans connotation axiologique, ces deux logiques économiques comme constitutives d'une même réalité économique : celle de l'économie de transactions marchandes et/car de transactions de direction. Nous verrons plus bas, avec l'étude de la transaction de répartition (rationing), que dans l'approche commonsienne un troisième principe d'économicité de souveraineté / citoyenneté / gouvernance, participant de la même réalité économique, s'ajoute et s'articule aux deux principes évoqués ci-dessus.

Dans sa spécificité propre, le principe d'économicité en termes d'efficacité productive / coopération technique / direction (management) gouvernant la transaction de direction s'applique particulièrement à la relation salariale sous la forme de la transaction de direction salariale. Dans cette dernière, selon l'approche commonsienne, et sans entrer cette fois dans l'analyse que permettrait cette dernière quant aux particularités du processus séquentiel (négociationnel, transactionnel, administratif) mais que Commons ne développe pas, les transacteurs sont nécessairement, car l'efficacité technique productive en dépend, dans un rapport de supérieur à inférieur. La relation salariale, dans le monde effectif de la production, repose sur une logique hiérarchique où le supérieur, c'est-à-dire le salarié plus haut placé dans l'ordre de la compétence technique reconnue formellement dans l'organigramme et ainsi de suite en amont jusqu'à la direction du going concern productif, commande à un inférieur, c'est-à-dire au salarié hiérarchiquement en aval, et ce dernier doit obéir, manifestant ainsi sa conduite coopérative et sa contribution à l'efficacité technique dans l'œuvre commune, conjointe et coordonnée hiérarchiquement, de la production. Le contenu de la transaction de direction salariale est donc un ensemble de règles opérantes (working rules) de conduite organisées, c'est-à-dire formelles et plus ou moins explicites, et 
inorganisées, c'est-à-dire plus ou moins informelles et fondées sur des pratiques habituelles, qui structurent l'action collective selon une logique coopérative faite de commande (command) et d'obéissance (obediance), d'ordres et d'exécutions, en somme de relations de subordination entre principal (supérieur) et agent (inférieur). Le principe de coopération régissant l'agence (agency) de production, principe dont Commons nous dit qu'il est une nécessité de l'ordre productif efficace, est donc pour lui, en régime de relations industrielles de type salarial, un principe fondé sur la subordination technique. En termes de futurité significative commune, la conception commonsienne de la transaction de direction se présente donc comme un monde institué de la production dans lequel les transacteurs voient leurs actions individuelles projetées [peut(pas), pourrait] et contrôlées [doit(pas)] selon une logique d'efficacité et de compétence techniques d'ensemble qui leur échappe pour une bonne part. Ces transacteurs, selon les situations et leurs positions dans les transactions de direction, c'est-à-dire (Tableau II) selon leur statut économique hiérarchique de sécurité / vulnérabilité et de liberté / conformité, selon les relations de (non)droit/(non)devoir qui s'imposent à eux, s'inscrivent dans un plan d'ensemble dans lequel ils sont plus ou moins convoqués à occuper des rôles productifs dont la cohérence d'ensemble et l'architecture hiérarchique se présentent à eux comme objectives, c'est-à-dire un monde du travail institué, qui l'était avant eux et qui le sera après eux.

Ainsi, selon l'approche institutionnaliste commonsienne, l'organisation productive est donc constituée de l'ensemble des contenus concrets de ces transactions de direction, c'est-à-dire de l'ensemble des rôles productifs hiérarchiques et coordonnés qu'occupent respectivement et pour un temps des personnalités instituées de trans-acteurs productifs. Et cette organisation est d'autant plus prégnante comme institution économique de la production que l'action collective organisée et inorganisée à l'œuvre dans les transactions de direction contrôle des action individuelles routinières (routines), répétitives (repetitions) et empreintes de similarités (similarities), que la sécurité des anticipations (security of expectation) et les hypothèses habituelles des transacteursproducteurs institués sont ancrées dans une futurité significative commune dont les dimensions de coopération hiérarchique et d'efficacité technique coordonnée sont établies et stables ${ }^{19}$.

Cependant, malgré cette conception de l'organisation productive qui pourrait laisser croire que cette dernière est totalement déterminée et fait des personnalités d'acteur institué dans les transactions de direction des automates de l'organisation, Commons considère deux ordres de faits, liés à l'initiative des

19 Pour une présentation de l'articulation conceptuelle entre organisation et institution chez Commons, voir L. Bazzoli \& V. Dutraive (2002). 
transacteurs individuels, qui concourent à rendre cette organisation évolutive. Le premier ordre de faits concerne, d'une part, le caractère inévitablement incomplet du contrôle de l'action collective dans les transactions de direction. La futurité significative commune des acteurs individuelles de ces transactions possède toujours des vacances, souvent aux frontières des règles opérantes de conduite inorganisée, qui sont autant d'espaces d'autonomie dans lesquels les salariés peuvent déployer à la fois leurs capacités de résistance à la subordination hiérarchique coercitive et leurs aptitudes à innover dans le sens de l'amélioration de leur bien-être au travail. D'autre part, l'inexistence parfois de facteurs complémentaires requis (compétences, moyens de production, etc.) pour le déploiement de l'action productive conduit certains transacteurs, subordonnés ou supérieurs, à initier une action stratégique (stategic action), c'est-àdire une action de type nouveau ou échappant (encore) au contrôle de l'action collective. Ces actions stratégiques ouvrent de nouvelles perspectives par rapport à la futurité significative commune routinière et donc de nouvelles possibilités de libération et d'expansion d'action (en particulier en faveur des supérieurs) ou d'action contrainte (en particulier au détriment des inférieurs). Le second ordre de faits qui risque de modifier l'organisation productive concerne le conflit et la remise en cause d'un pouvoir d'action (power of action), et d'une immunité (immunity) l'accompagnant, considérés, maintenant par rapport à antérieurement ou dans sa nouveauté, comme abusifs, c'est-à-dire causant pour certains acteurs individuels ou collectifs, en particulier les salariés subalternes, de nouvelles responsabilités (responsabilities) et incapacités (desabilities) quand ce n'est pas de nouvelles formes de coercition abusive. Ce dernier aspect, quant aux limites supportables par les salariés de la subordination requise techniquement par la coopération hiérarchique efficace, conduit à nouveau Commons à mettre en évidence la connexion économie/droit/éthique en matière de transaction de direction. La problématique du raisonnable (reasonabless) en cette matière concerne les limites du pouvoir managerial de direction quant à 1"usage" de la force de travail salariée. Il s'agit de raisonnablement délimiter la frontière entre, d'une part, l'incitation (inducement) acceptable et l'exercice d'un pouvoir de commandement technique légitime, d'autre part, la coercition et l'abus du pouvoir hiérarchique de direction. Cette problématique amène à nouveau Commons à analyser la question de l'autorité autorisée à autoriser les contenus nouveaux ou/et conflictuels de la transaction de direction salariale.

A cet égard, Commons reconnaît certes à certaines autorités internes au dispositif de direction managériale le pouvoir légitime d'exercer leur autorité d'autorisation de règles opérantes de conduite dans la transaction de direction. En particulier lorsqu'il s'agit de 
personnalités autorisées du fait de leur compétence technique et/ou leur aptitude reconnue par leurs subalternes. Cependant, il est aussi conscient que la dissymétrie des pouvoirs dans l'organisation productive en faveur de la hiérarchie managériale peut conduire à la subordination abusive et donc à la coercition inacceptable pour les salariés. Il est donc en faveur de l'existence d'instances d'autorisation sous forme législative, réglementaire, judiciaire, administrative (paritaire, de concertation, de conciliation, d'arbitrage, de négociation, etc.) interne (représentative, de participation, de règlement de conflit, etc.) ou externe (commission nationale, sectorielle, professionnelle, etc.) au going concern productif. Ces instances d'autorisation doivent garantir le caractère raisonnable de la subordination des salariés dans la transaction de direction salariale.

De plus, comme dans le cas de la transaction de marchandage salarial, et là aussi pour rééquilibrer les puissances respectives de détermination de la futurité significative commune des employeurs / directeurs et des salariés / subalternes, Commons recommande la reconnaissance légale d'un syndicalisme suffisamment puissant, quant à sa capacité à négocier et à peser dans les instances de concertation, arbitrage, conciliation, participation, etc., pour concourir à rendre la transaction de direction raisonnablement vivable pour les salariés et renforcer ainsi l'institution de la relation salariale. Et aussi, comme dans le cas de la transaction de marchandage, Commons considère que le raisonnable en matière de transaction de direction, en plus de devoir prendre en considération ce qui relève de la connexion économie/droit/éthique et dont les autorités d'autorisation sont redevables (argumentaires et justifications économiques, juridiques et éthiques), peut se concrétiser (dans les clauses "normatives" des conventions collectives par exemple) par l'alignement sur les "conditions de travail" les plus souhaitables et constatées dans d'autres going concerns productifs.

\section{3 - La transaction de répartition salariale}

Dans toute forme de société en devenir (going concern), que ce soit la nation dans son ensemble ou simplement une association, une entreprise, un syndicat, en somme un quelconque groupement humain, Commons constate qu'un principe de souveraineté (sovereignty) est présent pour assurer la gouvernance (government) et, dès lors, un statut de citoyenneté (citizenship) pour les membres du going concern. Plusieurs principes de souveraineté et régimes de gouvernance sont possibles dont, par exemple, le partage par vote (log-rolling), la coopération (coöperation), la négociation collective (collective bargaining), l'entente de commerce (trade agreement), l'arbitrage (arbitration), l'obligation (compulsory), la dictature 
(dictatorship), etc. Concernant l'activité économique, le principe de souveraineté se traduit alors, et selon le régime de gouvernance en vigueur, par une répartition (rationing) des charges (burdens) et des bénéfices (benefits) entre les citoyens du going concern. Cette répartition s'effectue soit en termes de fixation de quantités en nature, il s'agit alors d'une répartition en produit (output-rationing), soit en termes de fixation de prix monétaire, il s'agit alors d'une répartition par les prix (price-rationing). Ce sont ces aspects de l'activité économique que Commons étudie dans l'unité d'analyse qu'est la transaction de répartition (rationing transaction).

En ajoutant ce troisième type de transaction, la transaction de répartition concernant la souveraineté / gouvernance / citoyenneté (rationing), à la transaction de marchandage concernant la rareté / propriété / marchandage (bargaining) et à la transaction de direction concernant l'efficacité productive / coopération technique / direction (management), Commons pense épuiser avec ces trois unités d'analyse la variété des facettes réelles de l'activité économique. On retrouve donc ici, dans l'analyse institutionnaliste commonsienne, un traitement théorique intégré de la connexion économie / droit / éthique. Cette connexion est déclinée soit : en termes du triptyque traditionnel de l'économie politique - échange / transaction de marchandage, production / transaction de direction, répartition / transaction de répartition -; soit en termes du triptyque traditionnel du droit économique - droit de cession / transaction de marchandage, droit d'usage / transaction de direction, droit d'usufruit / transaction de répartition -; soit en termes du diptyque traditionnel de l'éthique économique - justice commutative / transaction de marchandage, justice distributive / transaction de répartition -, auquel Commons ajoute une justice que l'on pourrait appeler justice "productive" / transaction de direction. Sont donc ainsi couvertes analytiquement les trois dimensions traditionnellement étudiées de l'activité économique : le "marché" / transaction de marchandage, 1" organisation" / transaction de direction, la "(re)distribution" / transaction de répartition. Et cette couverture analytique s'applique aussi, chez Commons, spécifiquement à l'étude de la relation salariale et constitue, en quelque sorte, un découpage analytique du champ d'étude des relations industrielles.

En ce qui concerne la transaction de répartition salariale, comme dans les autres transactions de ce type, la relation entre les trans-acteurs en est une de supérieur à inférieur, de commande et d'obéissance une fois que les argumentations et plaidoiries ont été émises et que le régime de gouvernance s'est établi légitimement. Le détenteur ou l'instance de la souveraineté gouverne, selon le régime de légitimité en vigueur, en répartissant les charges et les bénéfices aux citoyens du going concern salarial. Concrètement, au niveau du 
going concern économique productif, de l'établissement salarial, l'employeur dispose de la souveraineté que lui confère sa légitimité inscrite : soit dans son pouvoir discrétionnaire reconnue en tant que propriétaire du capital ou actionnaire principal; soit dans une délégation de son conseil d'administration pour assurer la fonction exécutive; soit dans une convention collective (nationale, de branche, d'entreprise) ou autres accords (d'entreprise, d'instances intermédiaires, paritaires, etc.). Investi de cette souveraineté, l'employeur gouverne alors en répartissant les charges / prélèvements (contributions et cotisations à des assurances collectives, régimes et autres fonds, ainsi que toutes autres formes de retenues, imputations, etc.) et les bénéfices / avantages (rémunérations, primes; prestations des assurances collectives, régimes, fonds; intéressement, parts de participation, etc.). Ces charges et bénéfices sont pécuniaires et/ou en nature et les salariés y sont soumis en tant que citoyens sous la gouvernance de l'employeur. En somme, à ce niveau, les charges et les bénéfices salariaux concernent donc tous les aspects de traitement pécuniaire ou en nature attachés à la condition de salarié et dont l'employeur a la gouvernance du fait d'une souveraineté qui lui est reconnue de droit.

$\mathrm{Au}$ niveau le plus général du going concern de la collectivité (nationale, provinciale, etc.), la souveraineté dans la transaction de répartition salariale appartient maintenant aux pouvoirs publics. Cette souveraineté et la gouvernance qui l'accompagne trouvent leur légitimité dans le régime politique qui les fonde. Les autorités publiques agissent alors comme donneurs d'ordres aux membres des going concerns économiques, en particulier respectivement aux employeurs et aux salariés. Ceux-ci sont soumis à des charges sur le modèle : soit de l'astreinte fiscale pour toutes les sortes de "prélèvements obligatoires" (cotisations aux différents régimes d'assurance-sécutité sociale, chômage, accident-santé sécurité du travail, etc.); soit de la réglementation des prix et des revenus (fixation des salaires, salaire minimum, tarification des heures supplémentaires, pourcentage de masse salariale pour formation, logement, etc.). Quant aux bénéfices, ceux-ci sont répartis selon le modèle de 1"ayant droit" et suivant des logiques inscrites dans les politiques sociales, de sécurité du revenu, de l'emploi, de formation, de discrimination positive, d'équité salariale etc. En somme, la souveraineté du politique impose sa gouvernance sur tout un ensemble de composantes de la relation salariale et que l'approche institutionnaliste commonsienne analyse comme constitutifs de la transaction de répartition salariale. C'est à ce niveau, que s'exprime le plus clairement l'action régulatrice des pouvoirs publics sur la relation salariale. 
De plus, entre la gouvernance souveraine de l'employeur et celle des autorités publiques se situe aussi toute une variété d'instances intermédiaires souveraines dans ce domaine (mutualité, associations professionnelles, organisation d'économie communautaire, sociale, solidaire, etc.) ayant plus ou moins d'impact sur la transaction de répartition salariale selon leur degré de légitimité auprès des pouvoirs publics, des associations d'employeurs, des syndicats, etc. Ces instances intermédiaires œuvrent aussi dans le sens d'une régulation de la relation salariale.

Ainsi, comme pour les autres types de transaction salariale, la transaction de répartition salariale est fortement instituée. L'action collective s'y manifeste de façon particulière prégnante du fait même que nombre des règles opérantes de conduite qui contrôlent l'action individuelle des trans-acteurs échappent à ces derniers, s'imposent à eux comme des contraintes d'autant plus fortes qu'elles relèvent d'une souveraineté légitime éloignée de leur pouvoir d'action stratégique. Les employeurs mais surtout les salariés appréhendent cette action collective, en particulier tous les éléments de "couverture sociale" et de "redistribution des revenus" inscrits dans la transaction de répartition salariale, comme des faits objectifs qui structurent fortement leur futurité significative commune, qui participent significativement à déterminer leur statut de sécurité / vulnérabilité à venir. A tel point qu'un des aspects les moins contestés par les chercheurs en sciences sociales de la réalité de la construction sociale du fait salarial est celui de l'intégration (exclusion) sociale du (non)salarié de par son inscription ou non dans une transaction de répartition salariale. Une bonne part de la sécurité de la "condition salariale", et en conséquence de l'inclusion sociale, réside dans la possibilité pour les travailleurs de s'inscrire dans une futurité significative commune structurée par une transaction de répartition salariale dont l'autorité souveraine de gouvernance lui assure un avenir envisageable, un avenir sécurisé par une couverture sociale et une garantie de revenu. Dans cette optique, Commons a fortement contribué avec son analyse de la transaction de répartition, d'une part, à connecter la question "salariale" et la question de la "couverture sociale-revenu", c'est-àdire ce qui pourrait apparaître comme une séparation de 1"économique" et du "social" alors que ces deux dimensions de la réalité économique de la société salariale sont intimement liées et presque indissociables, d'autre part, à intégrer dans l'étude des relations industrielles l'une de ses composantes constitutives qu'est l'étude des "politiques sociales". Et à cet égard, la contribution de 
Commons à l'analyse et à la proposition de solutions raisonnables demeure d'une richesse intéressante ${ }^{20}$.

Dans la transaction de répartition salariale, la question de l'autorité autorisant les règles opérantes de conduite, c'est-à-dire les contenus de l'action collective en matière de répartition, est particulièrement importante. En effet, notamment du fait sa dimension régulatrice, la transaction de répartition instituée requiert une forte légitimité. Le type de souveraineté qui justifie la gouverne distributive dans cette transaction doit donc disposer d'une légitimité qui garantit sa puissance d'autorisation.

$\mathrm{Au}$ niveau élémentaire de la gouvernance du going concern économique, le conflit est toujours possible entre les citoyenssalariés gouvernés par le souverain-employeur quant à ce qui peut ou ne pas être autorisé en matière de règles opérantes de répartition. Dans le cas où l'employeur-souverain opterait unilatéralement pour un régime de gouvernance orienté vers une logique d'affaires (going business) légitimée par les intérêts propriétaires (proprietary interest) plutôt qu'orienté vers une logique industrielle (going plant) légitimée par les intérêts communs des salariés et de l'employeur, alors l'institution salariale risquerait d'être en difficulté. Les salariés pourraient remettre en cause cette orientation de la gouvernance et la souveraineté qui la fonde. La puissance d'autorisation des règles de répartition par la gouvernance d'affaires se heurterait à l'opposition des salariés prônant de leur côté une gouvernance plus en faveur des intérêts salariaux (emplois, salaires, avantages sociaux, etc.). Cette remise en cause de la répartition des charges et bénéfices au niveau du going concern productif nécessiterait, en situation de conflit ouvert, soit une renégociation entre salariés et employeur des règles de répartition, soit le recours à une instance extérieure et légitime autorisant ou non les règles de répartition en vigueur ou proposées respectivement par les parties en conflit.

Dans la situation de renégociation au niveau du going concern productif, et étant donnée la dissymétrie des puissances le plus souvent en défaveur des salariés, Commons recommande une présence légale syndicale suffisamment puissante pour négocier une entente sur les règles de répartition. Selon Commons, le cadre de la négociation collective et son résultat légalement formalisé par une convention collective sont la solution la plus raisonnable et/car la plus légitime. La souveraineté de la gouvernance de l'employeur, et son expression en termes de pouvoir d'autorisation des règles de répartition, se trouveraient ainsi légitimées et/car conformes à une futurité significative commune suffisamment soutenable et durable pour les salariés en matière de règles de répartition. L'exercice de la

${ }^{20}$ Pour une telle application de l'analyse commonsienne aux politiques sociales, voir S. Morel (2000). Sur l'influence de Commons en matière de politique sociale, voir J. D. Chasse (1994). 
souveraineté par la gouvernance de l'employeur dans le cadre d'une convention collective, c'est-à-dire d'une action collective organisée dans la transaction de répartition salariale, serait une garantie sociale raisonnable de la solidité et de l'inscription dans la durée de l'institution salariale.

Dans la situation où le conflit sur les règles de répartition ne pourrait pas être résolu par la signature d'une convention collective, Commons recommande l'intervention d'une instance légitime supérieure au going concen productif et ayant le pouvoir d'autoriser ou non les règles de répartition objets du conflit. En effet, si la souveraineté en matière de gouvernance de la transaction de répartition ne peut être obtenu au niveau conventionnel du going concern productif, cette souveraineté et son pouvoir de gouverner les règles de répartition doivent être recherchés à un niveau supérieur de souveraineté, celui d'instances légitimes, intermédiaires (paritaires, représentatives, sectorielles, corporatives, etc.) ou supérieures (législatives, réglementaires, judiciaires, etc.) d'administration de règlement de conflit. La vocation régulatrice de ces instances et l'existence institutionnelle d'une administration de règlement des conflits en matière de règles de répartition sont pour Commons, à défaut d'une résolution de conflit par la négociation collective, une condition nécessaire et raisonnable de la pérennité sociale de l'institution salariale.

C'est à l'occasion de ces renégociations ou de cette administration de règlement de conflit concernant les règles de répartition que ces dernières évoluent et concourent à transformer processuellement l'action collective de la transaction de répartition salariale, c'est-à-dire ce que seront pour les salariés et les employeurs leur futurité significative commune en matière de règles de répartition. De tels processus, aux niveaux local, intermédiaire ou supérieur, de négociation (conflictuelle, paritaire, partenariale, raisonnée, etc.) et d'administration/règlement de conflit (décisionnelle, arbitrale, conciliante, etc.), redéfinissent sans cesse les souverainetés et leur pouvoir de gouvernance ainsi que les autorités autorisant les règles de répartition. De plus, il est à noter que dans cette analyse commonsienne de la transaction de répartition salariale, comme d'ailleurs aussi dans ses analyses des transactions de marchandage et de direction salariales mais ici encore de façon encore plus marquée, les dimensions de régulation et d'évolution sont intimement imbriquées. De façon générale dans l'approche institutionnaliste commonsienne, les processus d'évolution des institutions sont fondamentalement des processus d'ajustement, plus ou moins conflictuels, de la régulation institutionnelle. 
Ainsi, comme nous avons tenté de le mettre en évidence, le cadre analytique de J. R. Commons offre une riche et utile "boîte à outils" pour l'étude des relations industrielles. Toutefois, une double difficulté semble jusqu'à présent s'opposer à l'opérationnalisation de ce cadre dans le champ d'étude des relations industrielles.

La première difficulté est d'ordre méthodologique ${ }^{21}$ et se présente essentiellement sous la forme de deux obstacles de taille. L'un de ces obstacles est l'hétérodoxie de la construction conceptuelle de l'analyse commonsienne. Celle-ci réside en premier lieu dans les fondements épistémologiques de cette analyse. Fondée sur la philosophie pragmatiste, la théorie institutionnaliste commonsienne de l'action et de l'institution économiques s'écarte significativement, sinon s'oppose, à l'orthodoxie "cartésienne" de la vision du monde social. Par exemple, comme nous l'avons vu à propos de la futurité significative commune qui guide les conduites économiques, les concepts d"action" et d"institution" ont un sens radicalement différent dans l'approche commonsienne par rapport à celui traditionnellement adopté dans les sciences sociales. En particulier, le principe de causalité institutionnelle de la futurité vers le présent, d'un troisième monde institué vers l'action individuelle ainsi sous contrôle, est totalement étranger à l'idée généralement admise, par analogie avec le monde physique, d'une causalité mécanique du passé vers le présent. L'hétérodoxie de l'analyse commonsienne réside aussi dans son mode de construction conceptuelle. Commons décline l'analyse de diverses facettes de son objet d'étude non en utilisant une méthode démonstrative abstraite hypothético-déductive ni en suivant une méthode empirique positiviste. Il pratique plutôt une méthode abductive, c'est-à-dire une méthode de circularité autocorrective entre induction et déduction ${ }^{22}$. Selon cette méthode, il produit alors des concepts pertinents mais dont la cohérence d'ensemble peut difficilement être présentée selon un ordre démonstratif linéaire. Ce n'est qu'une fois articulés les uns aux autres selon une configuration structurale que les concepts commonsiens apparaissent dans leur cohérence d'ensemble. Par exemple, son analyse des différents types de transaction, et de leurs formules respectives idéal-typiques et réel-typiques, se déploie comme une déclinaison des diverses facettes de l'activité économique régies selon des principes différents et dont la complémentarité rend compréhensible la totalité de cette activité. Enfin, toujours concernant l'hétérodoxie et la complexité de la construction conceptuelle de l'analyse commonsienne, et on pourrait encore en présenter de multiple autres aspects, l'accent mis par Commons sur

${ }^{21}$ Pour une discussion sur la méthodologie de Commons, voir M. H. Rutherford (1983) et Y. Ramstad (1995). Pour une lecture et une traduction structuralistes éclairantes de la méthode commonsienne, voir B. Théret (2001).

22 Sur cette méthode, voir M. R. Tool (1994). 
l'approche évolutionnaire et processuelle ainsi que sur l'inévitable normativité de la connaissance en matière de faits sociaux vont à l'encontre des canons des sciences sociales "normales". Alors que ces dernières, en particulier l'économie orthodoxe, sont le plus souvent statique, physicaliste et à prétention de neutralité axiologique, Commons assume pleinement dans ces constructions conceptuelles les caractères processuels, évolutionnaires et normatifs de l'activité économique. Par exemple, son analyse de la sélection artificielle des institutions met en évidence le concept d'autorité comme opérateur, en tant que sélecteur et en tant que personnalité ou instance, du processus d'évolution / (dé)(re)régulation institutionnelle de l'activité économique.

L'autre obstacle méthodologique qui nuit à l'approche institutionnaliste commonsienne est paradoxalement le succès de la méthode de l'étude de cas qui fait pourtant partie intégrante de l'analyse commonsienne. L'efficacité opérationnelle empirique de cette méthode quant aux résultats positifs et utilisables auxquels elle peut parvenir, et la production en ce domaine de Commons lui-même est impressionnante, cache la nécessité de la conceptualisation et de la théorisation comme étape supérieure de la production de savoir. C'est d'ailleurs à ce propos, et souvent par assimilation à la tradition de l'historisme allemand ou du positivisme sociologique, que l'institutionnalisme, et en particulier l'institutionnalisme commonsien est critiqué comme une approche faible en théorie. Cette critique jointe à celle d'hétérodoxie de la construction conceptuelle de l'analyse commonsienne comme sources de confusion, sinon d'inconsistance ou d'incohérence, scellent bien souvent le destin, c'est-à-dire le rejet, de l'approche commonsienne dans la "communauté" des chercheurs en sciences sociales, y compris dans le domaine des relations industrielles.

La seconde grande difficulté à laquelle se confronte l'approche commonsienne quant son opérationnalisation est sont caractère nécessairement transdisciplinaire. Ce dernier est systématiquement réaffirmé par Commons au travers de son rappel constant de l'exigence analytique de la prise en compte de la connexion entre économie, droit et éthique. Malheureusement, bien que le domaine d'étude des relations industrielles soit explicitement caractérisé par un objet spécifique, l'étude du monde du travail, et non comme une "discipline" et qu'en conséquence l'approche transdisciplinaire devrait donc s'imposer logiquement, il n'existe pas à ce jour un cadre analytique transdisciplinaire qui s'affirme dans le monde académique des "relations industrielles". Comme nous avons tenté de le montrer, le cadre d'analyse commonsien pourrait fournir un tel cadre. La tentative originelle allant dans ce sens, celle qui s'exprima au moment de la fondation du champ d'étude des relations industrielles par l'École du Wisconsin dans la lignée des travaux de Commons 
s'est avérée être manifestement un échec académique (Kaufman 1993). La tâche qui semble attendre les chercheurs convaincus de la pertinence de l'analyse commonsienne serait donc à la fois de mettre en évidence la fertilité du cadre analytique transdisciplinaire commonsien en matière d'étude des relations industrielles et de démontrer l'opérationnalité de ce cadre à propos de l'étude appliquée à des problèmes spécifiques du monde du travail ${ }^{23}$. Puisse cet article avoir modestement contribué au premier de ces objectifs ${ }^{24}$.

\section{Références bibliographiques}

ALBERT, A. \& RAMSTAD, Y. (1997) The Social Psychology Underpinnings of Commons's Institutional Economics : The Significance of Dewey's Human Nature and Conduct, Journal of Economic issues, 31(4), pp. 881-916.

ALBERT, A. \& RAMSTAD, Y. (1998) The Social Psychology Underpinnings of Commons's Institutional Economics II: The Concordance of George Herbert Mead's "Social Self" and John R. Commons's "Will”, Journal of Economic issues, 32(1), pp. 1-46.

AUSTIN, J. L. (1962) Quand dire, c'est faire, Paris, Seuil, 1991.

ATKINSON, G. \& REDD, M. (1992) The individual is a going concern, Journal of Economic issues, 26(2), pp. 469-476.

BARBACH, J. (1989) John R. Commons : pioneer of labor economics, Monthly Labor Review, 112(5), pp. 44-49.

BARBACH, J. (1994) Americanizing the labor problem: the Wisconsin School, dans C. Kerr \& P. D. Staudohar (eds) Labor Economics and Industrial Relations, Cambridge, Harvard UP, 1994, pp. 41-65.

BAZZOLI, L. (2000a) L'économie institutionnaliste du travail de J. R. Commons : un "pragmatisme en action", Cahiers du Gratice, $\mathrm{n}^{\circ}$. 19, pp. 101-134.

BAZZOLI, L. (2000b) L'économie politique de John R. Commons, Paris, L'Harmattan.

BAZZOLI, L. \& DUTRAIVE, V. (2002) L'entreprise comme organisation et comme institution : un regard à partir de l'institutionnalisme de J. R. Commons, Economie \& Institutions, $\mathrm{n}^{\mathrm{O}} .1$, $2^{\mathrm{è}}$ semestre, pp. 5-46.

${ }^{23}$ Voir à cet égard les articles de J. D. Chasse et de R. McIntyre \& Y. Ramstad dans ce numéro.

${ }^{24}$ Les articles dans ce numéro de $\mathrm{S}$. Morel et de B. Théret s'inscrivent aussi dans cette lignée. 
BAZZOLI, L. \& KIRAT, T. (1999) La relation entre le droit et l'économie dans les traditions institutionnalistes et post-coasienne : enjeux pour l'analyse de l'évolution, Economies et Sociétés, HS nº. 35, pp. 69-90.

BAZZOLI, L. \& KIRAT, T. \& VILLEVAL, M-C. (1994) Rules, contracts, and institutions in the wage-labor relationship: a return to institutionalism, Journal of Economic issues, 28(4), pp. 1137-1171.

BIDDLE, J. E. (1990) Purpose and evolution in Commons's institutionalism, Journal of Political Economy, 22(1), pp. 19-47.

CHASSE, J. D. (1991) The American Association for Labor Legislation, an episode in institutionalist policy analysis, Journal of Economic issues, 25(3), pp. 799-828.

CHASSE, J. D. (1994) The American Association for Labor Legislation and the institutionalist tradition in National Health Insurance, Journal of Economic issues, 28(4), pp. 1063-1090.

CHASSE, J. D. (1997) John R. Commons and the special interest issue: not really out of date, Journal of Economic issues, 31(4), pp. 933-949.

COMMONS, J. R. (dir.) (1905) Trade unionism and labor problems, Boston: Ginn.

COMMONS, J. R. (1909) American shoemakers, 1648-1895: a sketch of industrial evolution, Quarterly Journal of Economics, Nov. , pp. 3984.

COMMONS, J. R. (dir.) (1910-1911) Documentary history of American industrial society, Cleveland: A. H. Clark, 10 vol.

COMMONS, J. R. (1913) Labor and administration, New York: A. M. Kelley, 1964.

COMMONS, J. R. (1916) (avec J. B. Andrews), Principles of labor legislation, New York: A. M. Kelley, 1967.

COMMONS, J. R. (1918-1935) (en collaboration) History of labour in the United States, New York: Macmillan, 1918 : vol. 1 et 2, 1935 : vol. 3 et 4.

COMMONS, J. R. (1919) Industrial goodwill, New York: McGraw-Hill.

COMMONS, J. R. (1921) (en collaboration) Industrial government, New York: Macmillan.

COMMONS, J. R. (1924) Legal foundations of capitalism, New York: Macmillan.

COMMONS, J. R. (1934a) Institutional economics, New York: Macmillan. 
COMMONS, J. R. (1934b) Myself, New York, Macmillan.

COMMONS, J. R. (1935) The problem of correlating law, economics and ethics, trad. fr. par H. Beaujeaud : Le problème de la coordination du droit, de l'économie et de la morale, dans Recueil d'études sur les sources du droit, Paris, Sirey, 1935, T. III, 124-144.

COMMONS, J. R. (1950) The economics of collective action, [K. H. Parsons (ed)], Madison: University of Wisconsin Press, 1970.

COMMONS, J. R. (1996) John R. Commons: Selected Essays, [M. Rutherford \& W. J. Samuels (ed)], London, Routledge, 2 vol.

DELEDALLE, D. (1954) Histoire de la philosophie américaine, Paris, PUF

DEWEY, J. (1967- 1990) The Early Works, 1882-1898; The Middle Works, 1899-1924; The Later Works, 1925-1953, [J. A. Boydston ed.], Carbondale, Southern Illinois UP, 1967-1990: 36 vol.

DIGGINS, J. P. (1994) The Promise of Pragmatism, Chicago, The University of Chicago Press.

DUGGER, W. M. (1980) Property rights, law, and John R. Commons, dans W. Samuels (ed.) Institutional Economics, Aldershot, E. Elgar, 1988, vol. II, pp. 209-221.

DUTRAIVE, V. (1995) De l'analyse contemporaine des institutions aux institutionnalistes américains : un cheminement rétrospectif, dans T. Corei (1995) L'Economie Institutionnaliste, Paris, Economica, pp. 5-16.

GISLAIN, J-J. (1999) Les conceptions évolutionnaires de T. Veblen et J. R. Commons, Economies et Sociétés, Hors série HS no. 34, 1/1999, pp. 47-65.

GISLAIN J-J. (2000a) La naissance de l'institutionnalisme : Thorstein Veblen, dans A. Béraud \& G. Faccarello (dir) Nouvelle histoire de la pensée économique, Paris, La Découverte, Tome III, Chapitre XXX, pp. 74-115.

GISLAIN J-J. (2000b), L'artifice économique chez T. Veblen et J. R. Commons : surplus et rareté, pouvoir et ordre, Cahiers du GRATICE, no. 19, pp. 37-57.

GISLAIN, J-J. (2002) Causalité institutionnelle : la futurité chez J. R. Commons, Economie \& Institutions, $n^{\mathrm{O}} .1$, $2^{\mathrm{è}}$ semestre, pp. 47-66.

HILLS, S. M. (1998) The institutional theory of John R. Commons and its relevance to contemporary IR, in P. B. Voos (ed.) Industrial Relations Research Association Series, Madison, IRRA, vol. I, pp. 300307. 
HODGSON, G. M. (1998) The approach of institutional economics, Journal of Economic Literature, 36(1), pp. 166-192.

KAUFMAN, B. E. (1993)The origins \& evolution of the field of industrial relations in the United States, Ithaca: ILR Press.

KAUFMAN, B. E. (1998) John R. Commons : his contributions to the founding and early development of the field of personnal/HRM, in P. B. Voos (ed.) Industrial Relations Research Association Series, Madison, IRRA, vol. I, pp. 328-341.

MAUCOURANT, J. (1993) Au cœur de l'économie politique de la dette : l'approche de J. R. Commons, Revue du MAUSS, n. 2, pp. 209-218.

MAUCOURANT, J. (2001) L'institutionnalisme de Commons et la monnaie, Cahiers d'Economie Politique, nº. 40/41, pp. 253-284.

MEDEMA, S. G. (1998) Commons, Sovereignty and the legal basis of the economic system, dans W. Samuels (ed) The Founding of Institutional Economics, London, Routledge, pp. 97-114.

MIROWSKI, P. (1987) The philosophical bases of institutionalist economics, dans M. R. Tool (1988)(ed) Evolutionary economics, Armonk: Sharpse, vol. I, pp. 51-88.

MOREL, S. (2000) Les logiques de la réciprocité, Paris, PUF.

OLSON, M. (1966) Logique de l'action collective, Paris, PUF, 1978.

PEIRCE, C. S. (1879) Comment rendre nos idées claires, dans Textes anticartésiens, Paris Aubier, 1984, pp. 287-308.

PEIRCE C. S. (1931-1958) Collected Papers, Cambridge (Mass.), Harvard UP, 8 vol.

RAMSTAD, Y. (1986) A pragmatist's quest for holistic knowledge: the scientific methodology of John R. Commons, dans W. Samuels (1988) (ed.) Institutional Economics, Aldershot: E. Elgar, vol. II, pp. 138-176.

RAMSTAD, Y. (1990) The institutionalism of John R. Commons : theoretical foundations of a volitional economics, dans W.J. Samuels (ed) : Research in the history of economic thought and methodology, vol. 8, Greenwich: JAI Press, pp. 53-104.

RAMSTAD, Y. (1993) Institutional economics and the Dual Labor Market theory, dans M. R. Tool (ed.) (1993), Institutional Economics: Theory, Method, Policy, Boston, Kluwer Academic Publishers, pp.173231.

RAMSTAD, Y. (1994) On the Nature of Economic Evolution: John R. Commons and the Metaphor of Artificial Selection, in L. Magnusson (ed) Evolutionary and Neo-Schumpeterien Approachs to Economics, Dordrecht, Kluwer, 1994, pp. 65-121. 
RAMSTAD, Y. (1995), John R. Commons's puzzling inconsequentiality as an economic theorist, Journal of Economic Issues, 29(4), 991-1012.

RAMSTAD, Y. (1998) Commons's institutional economics : a foundation for the industrial relations field ?, in P. B. Voos (ed.) Industrial Relations Research Association Series, Madison, IRRA, vol. I, pp. 308-319.

RENAULT, M. (2000) Evolutionnisme et pragmatisme : Veblen, Dewey, Commons, Fondements philosophiques, Cahiers $d u$ GRATICE, no. 19, pp. 181-206.

RUTHERFORD, M. H. (1983) J. R. Commons's institutional economics, Journal of Economic Issues, 17(3), pp. 721-744; repris dans Samuels (1988-I), pp. 265-288.

RUTHERFORD, M. H. (1994) Institutions in Economics. The Old and the New Institutionalism, Cambridge, CUP.

THERET, B. (2001) Saisir les faits économiques : la méthode Commons, Cahiers d'Economie Politique, n. 40/41, pp. 79-137.

TOOL, M. R. (1994) An institutionalist mode of inquiry: limitations of orthodoxy, in P. A. Klein (ed) The Role of Economic Theory, Dordrecht, Kluwer, pp. 197-227.

WHALEN, C. J. (1993) Saving capitalism by making it good: the monetary economics of John R. Commons, Journal of Economic Issues, 27(4), pp. 1155-1179. 